\title{
Interplanetary scintillation observations of interaction regions in the solar wind
}

\author{
A. R. Breen ${ }^{1, *}$, P. J. Moran ${ }^{1}$, C. A. Varley ${ }^{1}$, W. P. Wilkinson ${ }^{1}$, P. J. S. Williams ${ }^{1}$, \\ W. A. Coles $^{2}$, A. Lecinski ${ }^{3}$, J. Markkanen ${ }^{4}$ \\ ${ }^{1}$ University of Wales, Aberystwyth, Wales, EU \\ ${ }^{2}$ University of California, San Diego, California, USA \\ ${ }^{3}$ National Centre for Atmospheric Research, Boulder, Colorado, USA \\ ${ }^{4}$ EISCAT Scientific Association, Sodankylä, Finland, EU
}

Received: 10 October 1997 / Revised: 2 February 1998 / Accepted: 4 February 1998

\begin{abstract}
Co-rotating interaction regions (CIRs) between fast and slow streams of plasma are a prominent feature of the solar wind. Measurements of interplanetary scintillation (IPS) using the three widely separated antennas of the EISCAT facility have been used to detect the compression regions at the leading edges of interaction regions and to determine the location and velocity of the structure. Observations show that interaction regions have developed as close to the Sun as 25-30 solar radii, a result supported by theoretical modelling which shows that the conditions needed for CIRs to develop exist inside 30 solar radii.
\end{abstract}

Key words. EISCAT · Interplanetary scintillation · Solar Wind

\section{Introduction}

Two-station measurements of interplanetary scintillation (IPS), in which simultaneous observations are made from widely separated sites (Armstrong and Coles, 1972), provide a powerful technique for determining the velocity of the solar wind at a wide range of latitudes and distances from the Sun (Rickett and Coles, 1991; Coles, 1995). The accuracy to which the velocity can be measured improves as the baseline between the sites increases (Bourgois et al., Breen et al., 1996a).

IPS measurements contain contributions from the whole ray-path from the source to the receivers, but as the "scintillation potential" - the ability of the medium

Correspondence to: Dr. A. R. Breen*

* Present address: Max-Planck Institut für Aeronomie, Postfach 20, D-37189 Katlenburg-Lindau, Germany Tel: +49 5556979 415; fax: + 495556979 410; e-mail: breen@helene.mpae.gwdg.de to produce scintillation - is proportional to $1 / R^{4}$ (where $R$ is the distance from the Sun), the observations are dominated by contributions from the portion of the raypath closest to the Sun. IPS observations are dominated by contributions from the portion of the ray-path (Fig 3), so the first estimate of velocity includes a $\cos (\theta)$ factor. If the location of fast and slow components of flow across the ray-path cannot be determined, then the only velocity estimate which can be quoted is the "apparent velocity", containing $\cos (\theta)$-weighted contributions from the whole of the line of sight (e.g. Breen et al., 1996b).

The first IPS observations from EISCAT used relatively short baselines between the sites and the results were interpreted as showing a single mean velocity with significant RMS variations parallel to and perpendicular to the direction of flow. However, in 1993 a series of observations was made in connection with the climb to high latitudes of the Ulysses spacecraft, and the geometry required by these observations demanded much longer baselines than had hitherto been used. The cross-correlation functions between scintillations received at the two sites in these observations showed two clear peaks, corresponding to distinct fast and slow velocities across the ray-path. Subsequent analysis of the EISCAT data set using a two-dimensional weak scattering model (Klinglesmith, 1997) showed that observations were best interpreted in terms of a solar wind containing two distinct components - a fast wind at $700-800 \mathrm{~km} \mathrm{~s}^{-1}$ and a denser slow wind at 300-400 km s${ }^{-1}$ (Grall et al., 1996; Breen et al., 1996b), a result consistent with measurements made by the particle instrument on Ulysses (Phillips et al., 1994).

Provided that the scattering volume is sufficiently far from the Sun for the phase variations introduced by irregularities to be small ("weak scattering"), then the scattering process can be treated as a succession of thinscreen events. The scintillation pattern observed at the receivers can then be assumed to be the sum of the patterns produced by each scattering event along the ray-path. Under these conditions, provided that regions 
of fast and slow wind can be located in the ray-path, it is possible to distinguish the contributions to the observed scintillation pattern made by fast and slow streams.

EISCAT observations are generally in weak scattering outside $14-15$ solar radii $(R)$ in the fast wind and $23-$ $30 R$ for the denser slow wind (systems such as MERLIN and the VLBA, which observe at higher frequencies, can penetrate even closer to the Sun).

Measurements from spacecraft (e.g. Snyder and Neugebauer, 1966; Krieger et al., 1973; Neupert and Pizzo, 1974; Nolte et al., 1977) have shown that the fast solar wind is clearly associated with coronal holes, so white-light or soft X-ray images of the corona can be used to locate the source regions of the fast wind (e.g. Snyder and Neugebauer, 1966; Schwenn, 1990; Grall et al., 1996). By projecting the IPS ray-path down to the corona the portions immersed in fast flow can be identified, allowing the velocities, densities and locations of the fast and slow winds across the ray-path to be determined accurately, independently and separately (Breen et al., 1996b, 1997).

Figure 1 shows the white-light intensity maps of the corona at $1.7 R$ calculated from measurements made by the HAO Mk.3 coronagraph on Mauna Loa. The white line overlaid on the plot is the IPS ray-path projected down to $1.7 R$ with an assumed velocity of $750 \mathrm{~km} \mathrm{~s}^{-1}$ (Fig 1a) or $325 \mathrm{~km} \mathrm{~s}^{-1}$ (Fig 1b). The triangle marks the closest passage of the ray-path to the Sun and the crossed circle the position of the Earth. The dots are at intervals of $10^{\circ}$ arc along the ray-path, corresponding to $10^{\circ}$ of latitude when projected down onto the corona. The correlation functions calculated from the IPS observations corresponding to the white-light maps are shown in Fig. 2 a, b In each case the average of the autocorrelation functions observed at the two sites and the observed cross-correlation function are shown as broken lines. The solid lines denote the auto- and crosscorrelation functions calculated using a two-dimension-
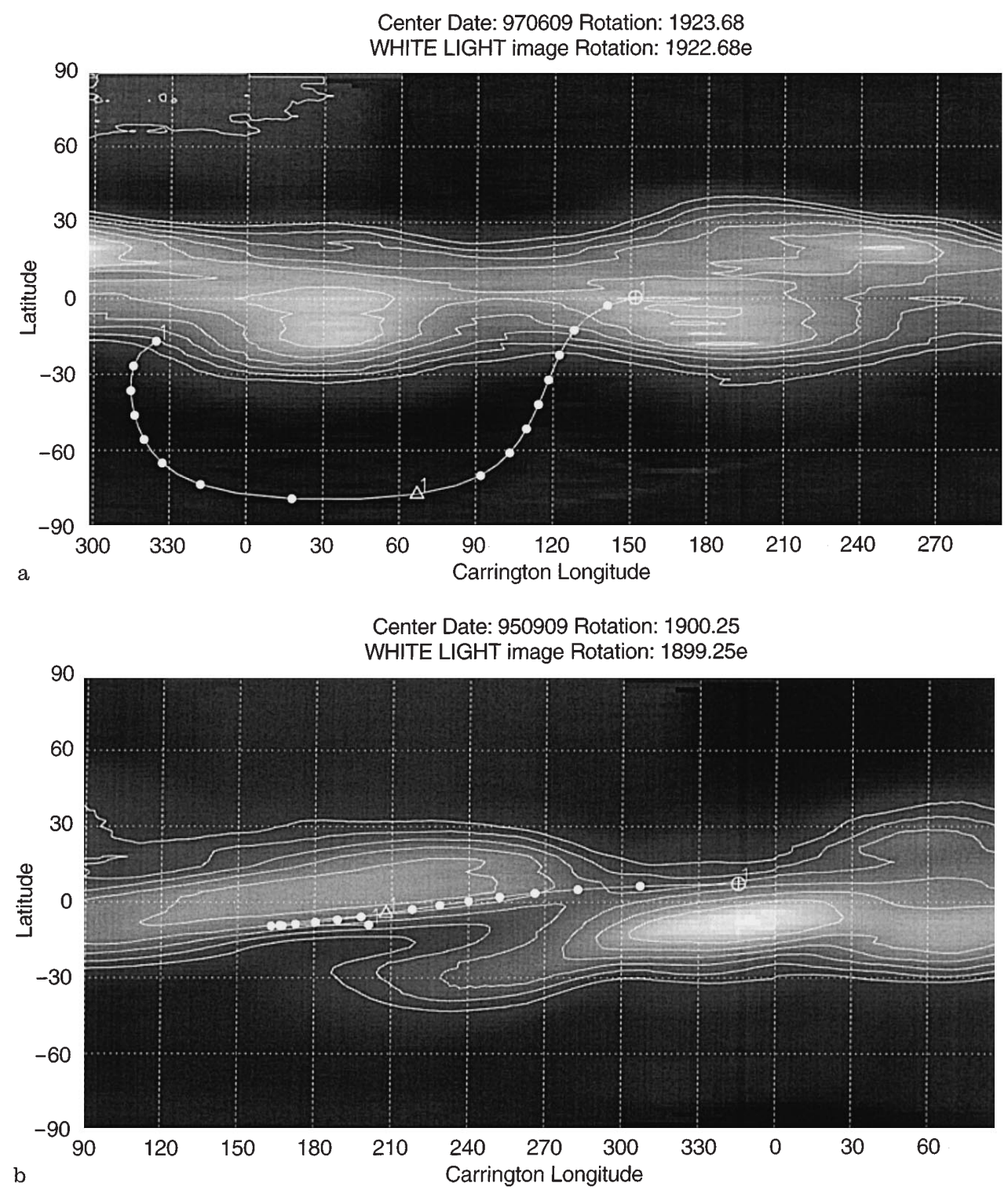

Fig. 1. a Map of white-light intensity at $1.7 R$, calculated from HAO Mk. 3 coronagraph measurements centred on 9 June 1997. Line 1 represents the IPS ray-path for measurements of the radio source $0521+166$ on 9 June 1997 , projected down to $1.7 R$ with an assumed velocity of $750 \mathrm{~km} \mathrm{~s}^{-1}$. The triangle marks the closest approach of the ray-path to the Sun and the crossed circle denotes the position of the Earth. Almost all of the ray-path lies above the southern polar coronal hole. b Map of white-light intensity at 1.7 $R$ centred on 9 September 1995. Line 1 represents the IPS ray-path for measurements of the radio source $1150-003$ on 9 September 1995 , projected down to $1.7 R$ with an assumed velocity of $325 \mathrm{~km} \mathrm{~s}^{-1}$. The whole of the raypath lies above the bright equatorial corona 


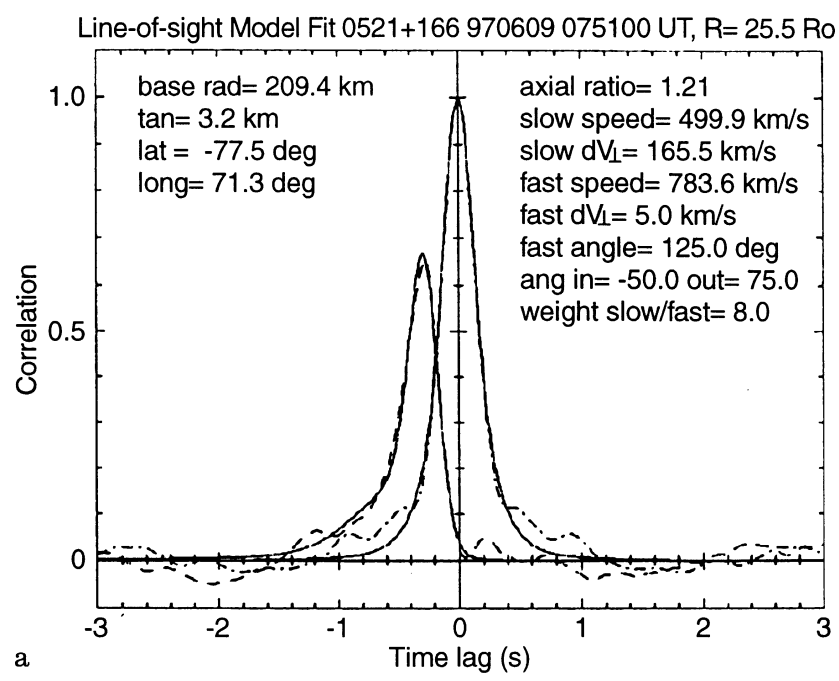

Fig. 2. a Auto- and cross-correlation functions for the observation of $0521+166$ on 9 June 1997. The broken lines denote the measured correlation functions and the solid lines the results of fitting using a 2D

al weak scattering model that calculates the final correlation function as the sum of scattering events taking place on $5^{\circ}$ segments of the ray-path (Fig 3). Provided that the whole of the ray-path lies in the weak scattering regime this model can be used to provide accurate and independent estimates of fast- and slowstream velocities and relative scattering potential. There are still some difficulties in determining the exact position of the fast streams, as the expansion of the solar wind is known to be significantly super-radial inside about $5 R$. However, comparison of the Mk.3 maps (at $1.7 R$ ) with maps derived from LASCO C2 data at $5 R$ indicate that the over-expansion of the fast wind is relatively small in the azimuthal direction, though more significant meridionally. For a typical IPS observation for which the closest approach of the ray-path to

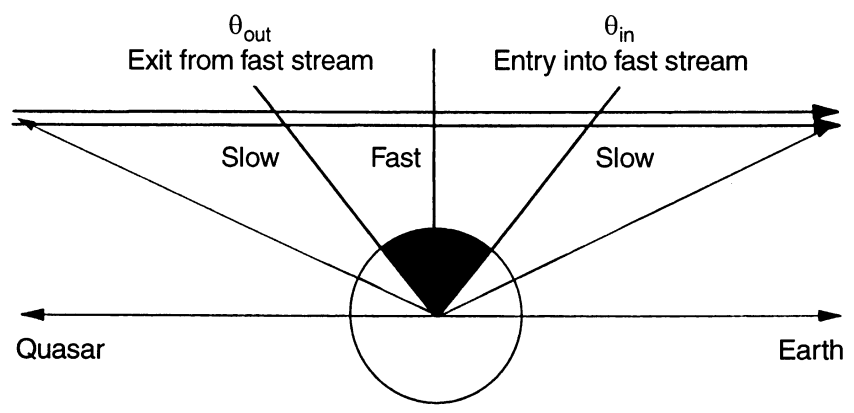

Fig. 3. Schematic diagram of IPS observations, showing the method used to de-convolve the contributions from different parts of the raypath. The ray-path enters the fast stream when the angle along the ray-path is $\theta_{\text {in }}$ and leaves it at $\theta_{\text {out }}$, where $0^{\circ}$ angle is the point at which the ray-path is closest to the Sun. The scattering of radio waves from the quasar is considered as a series of thin-screen events, with the scattering parameters calculated every $5^{\circ}$ along the ray-path. Fast and slow streams are treated separately, so the integration is performed for (1) distant slow stream $\left(\theta: 90^{\circ} \geq \theta>\right.$ out $)$, (2) fast stream $\left(\theta_{\text {out }} \theta>\right.$ in $)$ and local slow stream $\left(\theta_{\text {in }} \geq \theta>-90^{\circ}\right)$

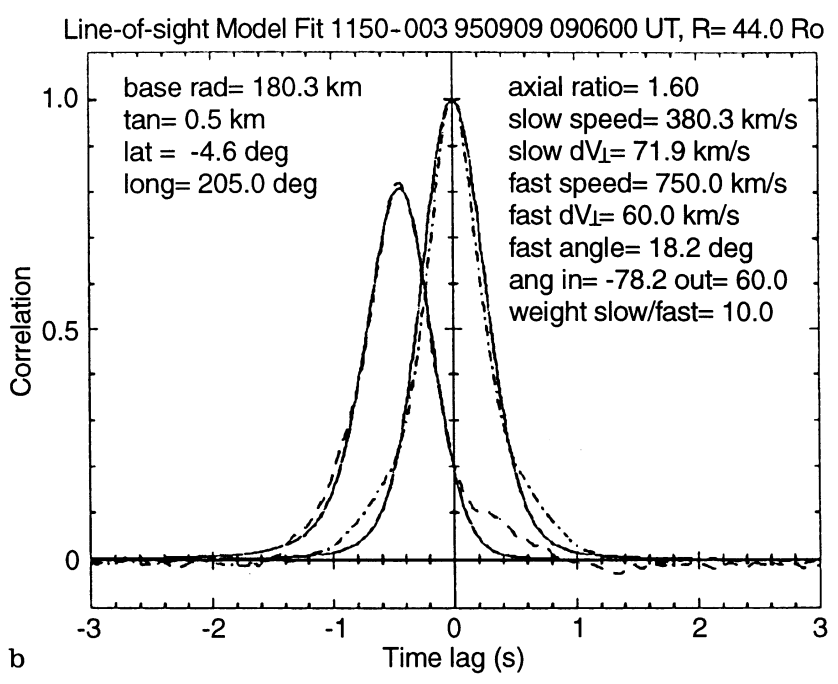

weak scattering model. The observation in dominated by fast flow. b Auto- and cross-correlation functions for the observation of 1150003 on 9 September 1995. The observation is dominated by slow flow

the Sun lies at $30^{\circ}$ latitude, the uncertainty in the angle of the ray-path immersed in fast flow will be $10^{\circ}-15^{\circ}$ Uncertainties of this order do not have a significant effect on the velocities derived from the analysis, although they can introduce errors in the estimates of relative scattering potential. In general, observations at lower latitudes are affected less by the continued expansion of fast streams (as the ray-path lies at almost constant latitude, where the effects of over-expansion are small).

Figure 4a shows the white-light map corresponding to an observation in which the ray-path passed from a region above the bright, "non-hole" corona to a region above a coronal hole and back. By associating the bright corona with the slow solar wind and the coronal hole with fast flow we can estimate that the IPS ray-path is immersed in the fast wind over $40^{\circ}$ of arc from its closest approach to the Sun and in the denser slow wind for the remainder of its length. This allows the scattering events taking place in the fast and slow winds to be calculated separately and the effects of distance from the Sun and the reduction of the apparent velocity by $\cos (\theta)$ to be taken into account. The observed and modelled correlation functions are shown in Fig. 4 b: the agreement is very good, while the velocities of the fast and slow streams (at 841 and $458 \mathrm{~km} \mathrm{~s}^{-1}$, respectively) are consistent with observations clearly dominated by fast and slow streams.

The velocity distribution for 245 EISCAT IPS observations made between May 1995 and July 1997 is shown in Fig 5. The distribution is clearly dominated by fast $\left(750-800 \mathrm{~km} \mathrm{~s}^{-1}\right)$ and slow $\left(300-400 \mathrm{~km} \mathrm{~s}^{-1}\right)$ streams, as discussed in Breen et al. (1996b) but there are also a number of points at $500-600 \mathrm{~km} \mathrm{~s}^{-1}$. By selecting only streams that are clearly dominated by flow from above either the bright corona or from above coronal holes it is possible to determine whether these two populations do indeed have different characteristics 

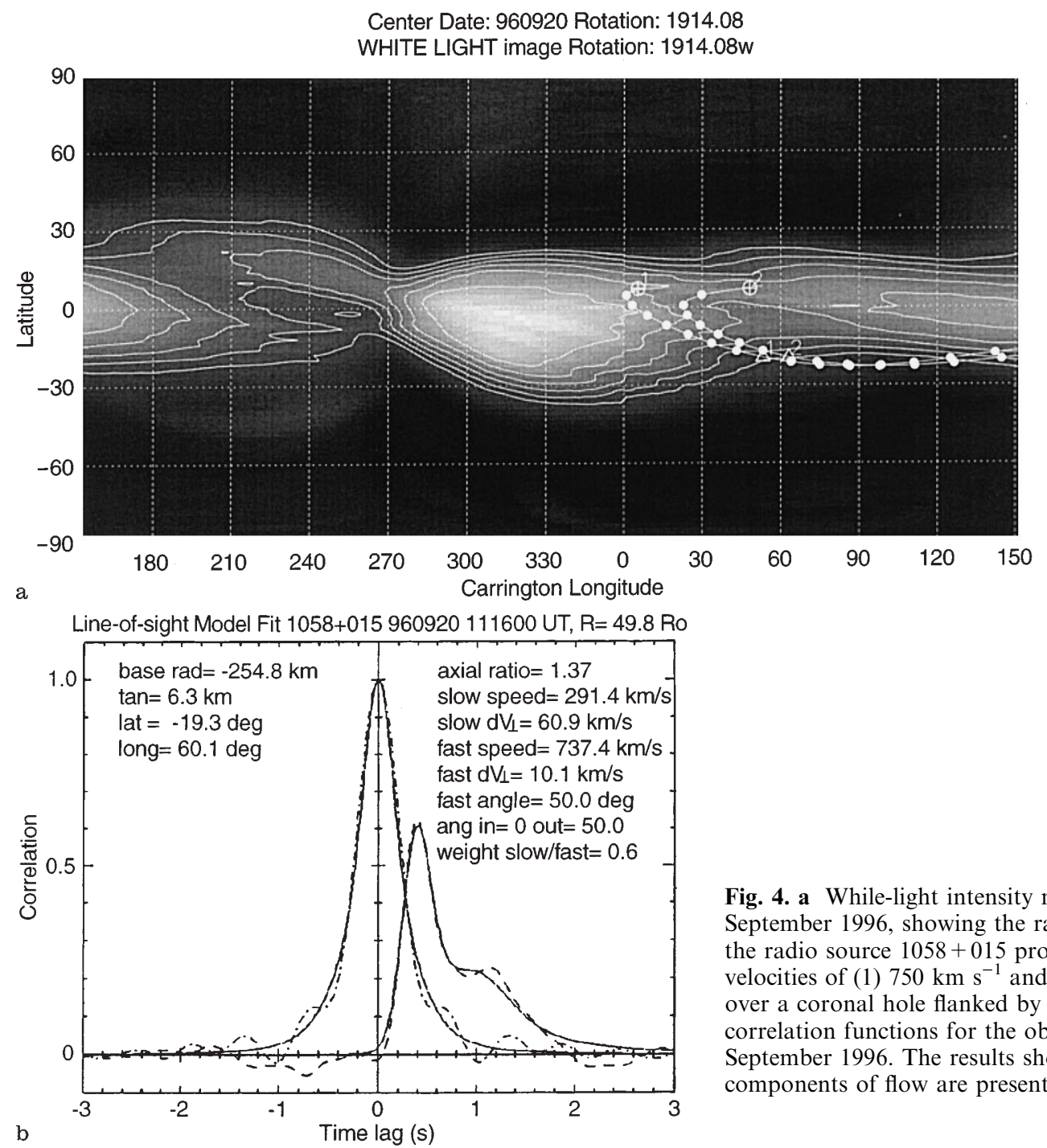

Fig. 4. a While-light intensity map of the corona at $1.7 R$ on 9 September 1996, showing the ray-path for EISCAT observations of the radio source $1058+015$ projected down to $1.7 R$ with assumed velocities of (1) $750 \mathrm{~km} \mathrm{~s}^{-1}$ and (2) $325 \mathrm{~km} \mathrm{~s}^{-1}$. The ray-path passes over a coronal hole flanked by bright regions. b Auto- and crosscorrelation functions for the observation of $1058+015$ on 9 September 1996. The results show that distinct fast and slow components of flow are present

and whether intermediate velocities exist. Figure 6 shows the velocity distribution for observations for which more than $97.5 \%$ of the observed scintillation is expected to come from above either the bright corona or

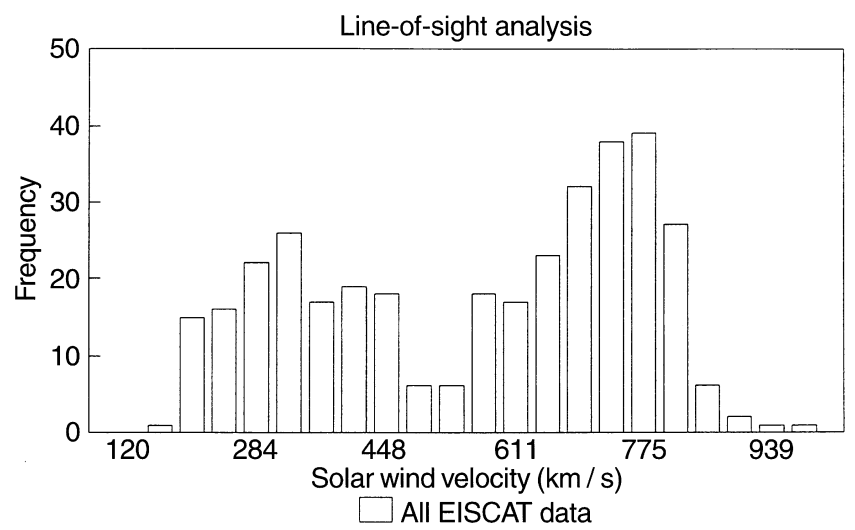

Fig. 5. Histogram showing the velocity distribution of the solar wind, as measured by EISCAT between April 1995 and July 1997 from above coronal holes. It is immediately clear from this distribution that - as expected - fast flow is associated with coronal holes and slow flow with the "non-hole" corona. Furthermore, the fast and slow streams are well defined with no overlap. The means and standard deviations of the fast and slow-stream velocities remained remarkably constant when streams contributing between $>99 \%$ and $>80 \%$ of the scintillation were considered (Table 1). This allows us to speak with confidence of a fast solar wind with a velocity close to $750 \pm 70 \mathrm{~km} \mathrm{~s}^{-1}$ that originates in coronal holes and a slow wind, found above the bright corona, with a velocity close to $325 \pm 80 \mathrm{~km} \mathrm{~s}^{-1}$. As discussed by Grall et al. (1996) IPS velocities will be biased slightly above the true background flow velocity by the effect of Alfvén waves propagating through a non-homogenous medium. The degree of bias introduced is difficult to determine exactly (although Grall et al. showed that the maximum possible value would always be less than $200 \mathrm{~km} \mathrm{~s}^{-1}$ ), but the close agreement of EISCAT observations with Ulysses measurements suggests that the effect is small. Taken together, these results provide 


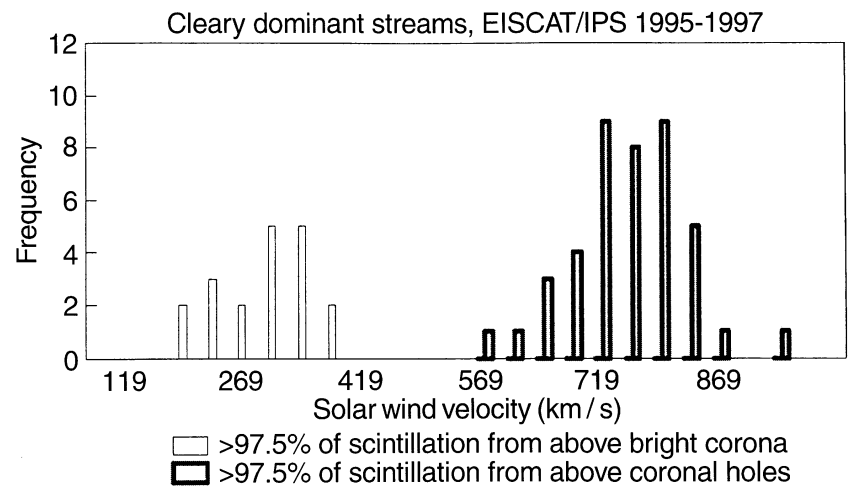

Fig. 6. Velocity distribution of clearly dominant streams (contributing $>97.5 \%$ of observed scintillation), 1995-1997

strong support for the central assumption made in analysing the observations shown as Figs. 1, 2 and $4-$ that white-light maps of the corona may be used as a guide to the location of fast and slow streams of solar wind.

We characterise intermediate velocities as those falling between $\left(V_{\text {slow }}\right)+2 \sigma\left(V_{\text {slow }}\right)$ and $\left(V_{\text {fast }}\right)-2 \sigma$ $\left(V_{\text {fast }}\right)$. The data set contains 25 observations of intermediate velocities. Four of these observations were made during the passage of a coronal mass ejection (CME) on 13 September 1996 (Breen et al., 1997), leaving 21 observations of intermediate velocities that are not associated with any obvious transient event.

In two earlier paper (Breen et al. 1996b, 1997) we hypothesised that these intermediate velocities corresponded to observations of regions of interaction between fast and slow streams and - as such represented the early stages of development of to corotating interaction regions observed by spacecraft at $0.3 \mathrm{AU}$ and beyond.

\section{Co-rotating interaction regions in the solar wind}

Co-rotating fast streams in the solar wind were first observed in Mariner 2 data in 1962 (Snyder and Neugebauer, 1966). It was soon realised that the presence of two distinct velocities at the same solar latitude would lead to discontinuities in the flow as the Sun rotates (see Fig. 3.10 in Schwenn, 1990), and these "stream interfaces" were defined by Burlaga (1974) as boundaries characterised by a decrease in density by a factor of $\sim 2$ over a distance of $<10^{6} \mathrm{~km}$, accompanied by a similar increase in kinetic temperature and a speed increase.

At the leading edge of a fast stream the rotation of the Sun carries fast flow (from a coronal hole) under slow flow from the same latitude and a more easterly longitude. As the magnetic fields carried by the two streams prevent the flows from penetrating each other, a compression region forms at the boundary between the two streams. This region is characterised by plasma densities higher than either of the undisturbed streams and by velocities intermediate between those of the fast and slow solar winds (Gosling et al., 1972, 1978). At the trailing edge of the fast stream the spirals of magnetic field diverge, producing a region of lower density and intermediate velocity (Sarabhai, 1963; Gosling et al., 1972) These compression and rarefaction region are now familiar from spacecraft observation from 0.35-5.0 AU (70-1100 R).

The intensity of the interaction increases with distance from the Sun, as the angle between the spirals of magnetic field carried by the fast and slow streams steepens. Close to the Sun the angle is small and the characteristic speed with which pressure signals propagate is high (Gosling et al., 1972), limiting the resultant flow deflection and density enhancement to narrow longitude ranges. Helios observations showed that CIRs typically extended over $3-6^{\circ}$ of longitude at $75 R$ and that the total longitudinal range affected by compression - the "co-rotating interaction region" or CIR - is typically about $30^{\circ}$ wide at $215 R$, although the flux tubes affected may cover as much as $70^{\circ}$ of longitude (Schwenn, 1990) At greater distances from the Sun (beyond $90 R$ ) the angle between the streams increases and the longitudinal extent of the interaction region grows rapidly, reaching up to $70^{\circ}$ by $215 R$. Helios observations showed that CIRs tended to evolve from abrupt, "mesa-like" features to broader, less steep structures as distance from the Sun increased (Schwenn et al., 1976).

Between 70 and $215 R$ longitudinal broadening of the CIR offsets the effects of kinematic steepening, but at greater distances the velocity gradients become steeper again, culminating in the formation of co-rotating shocks at the boundaries of the compression region at $215 R$ and beyond (e.g. Wimmer-Schweingruber et al., 1997). At the distances from the Sun accessible to EISCAT (15-120 R) the angle between the streams is too shallow for such dramatic effects to be observed, although by $30 R$

Table 1. Clearly dominant fast and slow streams in EISCAT IPS data

\begin{tabular}{|c|c|c|c|c|}
\hline $\begin{array}{l}\text { Minimum acceptable } \\
\text { stream weight } \%\end{array}$ & no. fast streams & $V_{\text {fast }} \mathrm{km} \mathrm{s}^{-1}$ & no. slow streams & $V_{\text {slow }} \mathrm{km} \mathrm{s}^{-1}$ \\
\hline 0.990 & 22 & $729 \pm 63$ & 19 & $363 \pm 63$ \\
\hline 0.950 & 52 & $748 \pm 73$ & 32 & $325 \pm 76$ \\
\hline 0.900 & 80 & $750 \pm 80$ & 49 & $329 \pm 77$ \\
\hline 0.850 & 90 & $749 \pm 77$ & 64 & $324 \pm 74$ \\
\hline
\end{tabular}


conditions do allow a considerable degree of steepening in the interaction region, as discussed in Sect. 4.

MHD modelling (e.g. Pizzo, 1989; Hu, 1993) has shown that significant enhancements in solar-wind density ahead of the stream interface can be produced by $65 R$, even when the source-surface for the interaction is assumed to lie at $32 \mathrm{R}$. These models have also demonstrated that the intensity of the interaction decreases as the angle between the stream interface and the ecliptic plane increases (Pizzo, 1991).

IPS observations are highly sensitive to conditions in the compression region of a CIR, as the scintillation potential is proportional to the square of the solar- wind density. A narrow compression region can therefore produce a detectable effect in the correlation functions derived from IPS measurements. The low-density divergence region at the trailing edge of the CIR - being more tenuous than either of the undisturbed streams - is not likely to contribute any detectable signature under normal circumstances.

\section{Observations}

\subsection{Observations of CIRs}

The data used in this study came from IPS campaigns carried out at EISCAT in the summers of 1991-1997. 245 observations made from April to October in 1995 and 1996 and April to July in 1997 have been analysed using the two-dimensional ray-path integration model described in Sect. 1, the boundaries of the fast and slow streams having been deduced from white-light coronal maps. The same analysis method has been applied to observations of particular interest from earlier years.

CIRs are formed when a fast stream is carried under slow wind from the same latitude and from a more easterly longitude. IPS observations are insensitive to the rarefaction regions at the trailing edge of CIRs, so IPS observations may be expected to show evidence of interaction regions when the ray-path passes from slow flow into fast flow as almost constant heliographic latitude, the fast stream lying to the west of the slow stream. The schematic diagram in Fig. 7 shows four possible geometries where the IPS ray-path passes through regions of fast and slow flow: in observation 1 the ray-path passes from slow flow, into fast flow and back to slow flow at almost constant latitude. In this case we would expect a compression region to form at the easterly boundary of the fast stream. In observation 2 the ray-path lies in fast flow at its westerly end and in slow flow for the remainder of its path, so we would expect a compression region to form between the streams at the easterly end of the ray-path. Observation 3 reverses the geometry of 2, with the slow stream lying to the west of the fast stream. In this case the rarefaction region lies in the IPS line of sight but is most unlikely to contribute enough scintillation to be detectable. We would expect this observation to show the signature of undisturbed fast and slow streams of solar wind. Finally, in observation 4 , the fast stream lies to the
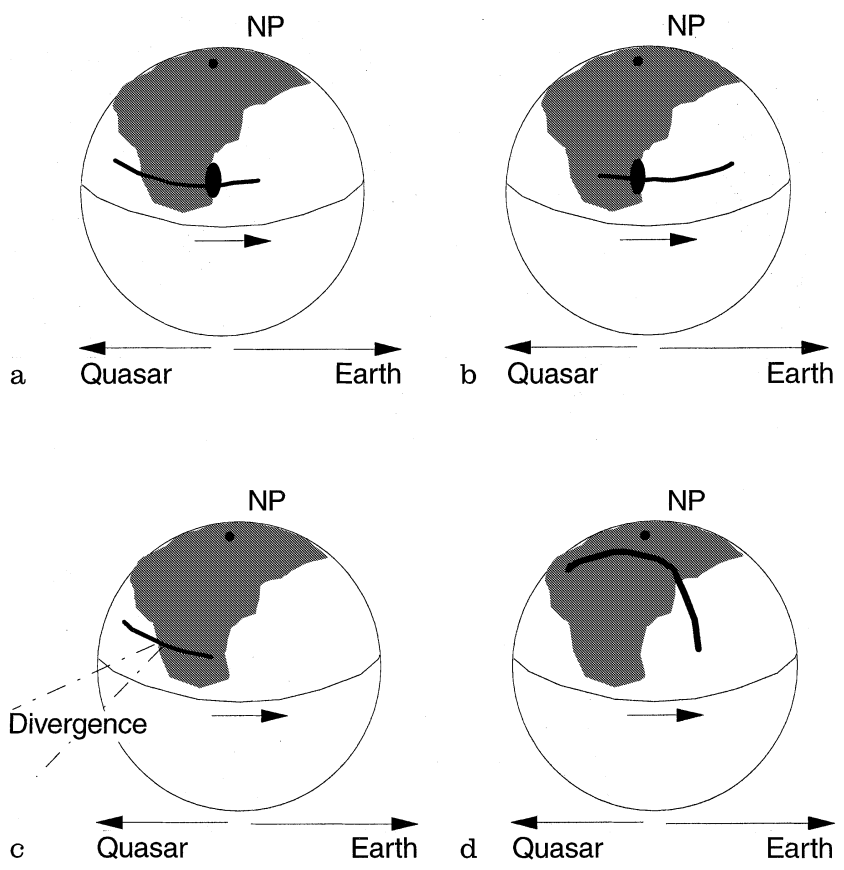

Fig. 7a-d. Schematic diagrams showing different geometries for IPS observations of fast and slow streams. The view is looking down on the Sun from the north-west. The arrow denotes the direction of rotation of the Sun, the shaded area represents a coronal hole and the heavy dark line the IPS ray-path projected down onto the disc of the Sun. Black areas represent the anticipated location in the ray-path of compression regions. In a fast flow lies at a higher Carrington longitude than slow flow, and will overtake it as it expands out from the Sun, producing a compression region. The same is true in $\mathbf{b}$, but in $\mathbf{c}$ the fast flow lies at a lower longitude and will diverge from the slow wind. In $\mathbf{d}$ the fast flow lies at a higher latitude than the slow flow, so the streams flow past each other without producing a compression region

west of the slow stream but at a higher latitude. There may be a small degree of compression due to the slightly super-radial expansion of the fast wind discussed by Moran et al. (1998), but the velocity differences involved are an order of magnitude lower than those resulting from solar rotation. In this case the fast and slow winds flow past each other and do not interact and the IPS observations should show two undisturbed streams.

An example of a clear IPS observation of a CIR is given in Fig. 8. In this case the IMP-8 satellite (at $215 R$ ) was able to provide velocity measurements that confirmed the existence of a CIR and stream interface at exactly the position expected from the coronal maps. Figure 8a shows the white-light derived from HighAltitude Observatory measurements centred on 5 September 1991. The superimposed lines are the ray-path for the EISCAT observation of the strong radio source $1229+020$ on 5 September 1991, projected down to the corona with assumed velocities of 800 (line 1) and $400 \mathrm{~km} \mathrm{~s}^{-1}$ (line 2). Both projections take the ray-path across the boundary of a low-latitude coronal hole, with the sector above the hole extending over an angle from $+10^{\circ}$ to $-25^{\circ}$, where negative angles are towards the Earth (see Fig. 3 for an explanation of the notation). The main region of fast flow is nearer to the Earth than 

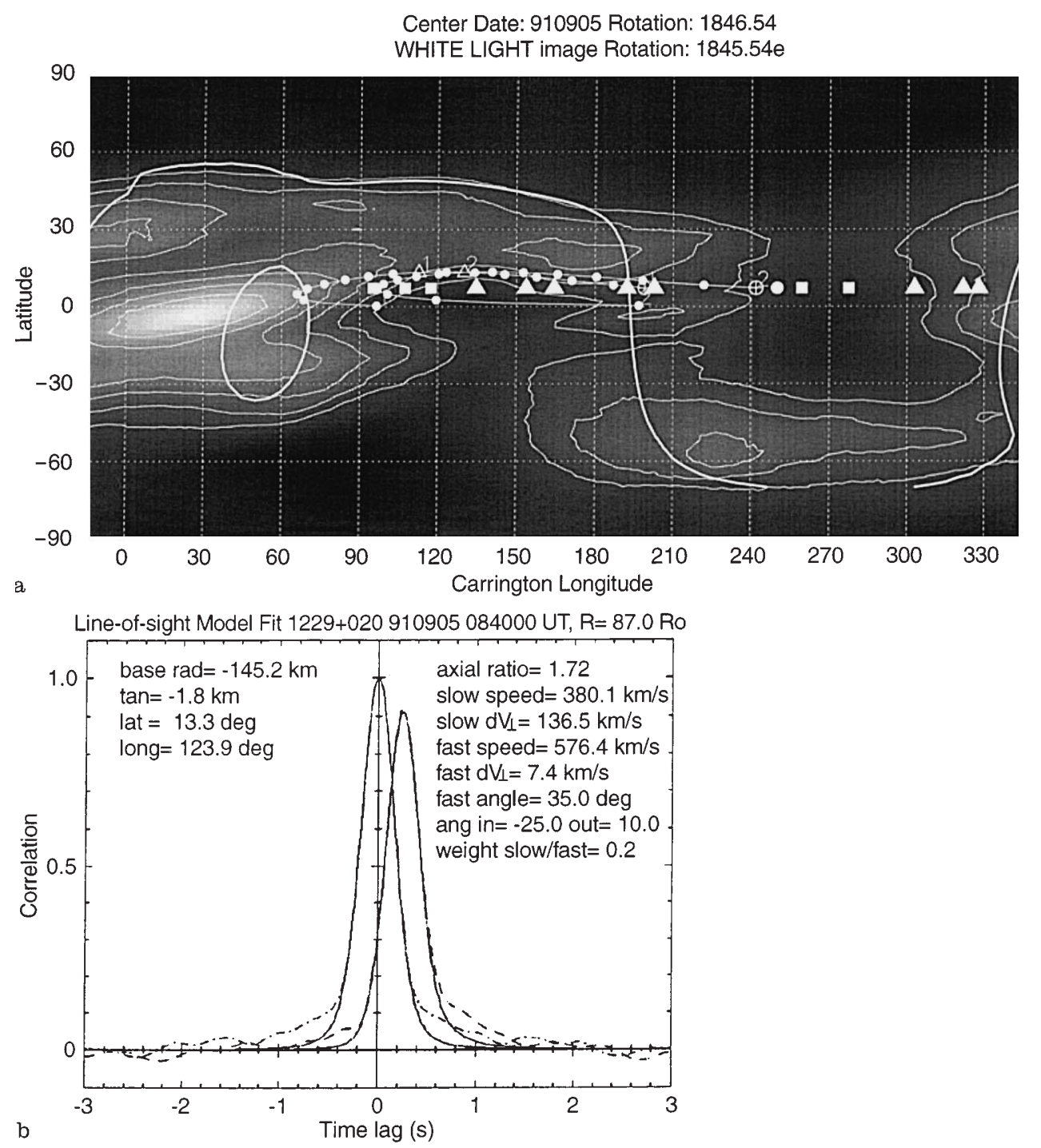

Fig. 8. a White-light map showing the ray-path for an EISCAT observation of $1229+020$ on 5 September 1991, projected down to 1.7 $R$ with assumed velocities of $750 \mathrm{~km} \mathrm{~s}^{-1}$ (line 1) and $325 \mathrm{~km} \mathrm{~s}^{-1}$ (line 2 ). The white line stretching across the whole of the map is the magnetic equator of the Sun, calculated from Stanford magnetic data. The white triangles mark regions of intermediate-velocity flow, measured by the IMP- 8 spacecraft at $215 R$ as the region observed

the slow flow, which on the west limb of the Sun will carry the fast flow under the slow region as the Sun rotates. The geometry indicates that the fast stream is overtaking a region of slow flow.

Figure $8 \mathrm{~b}$ shows the auto- and cross-correlation functions of the scintillations observed by the EISCAT sites at Kiruna and Sodankylä during the observation corresponding to Fig. 8a. The dotted lines denote the average auto-correlation function of the scintillations observed at each site and the cross-correlation function of the scintillations observed at the two sites, with the solid lines showing the results of the two-dimensional scattering model. The fit of the model to the data is extremely good, showing a dominant "fast" stream and a slow tail to the cross-correlation function( the bulge at longer lags). Both components are clearly visible in the

by IPS rotated past the Earth. b EISCAT correlation functions for the observation shown in a. The observed correlation functions are shown as broken lines and the results of the model fit as solid lines. The "fast" component is slower and produces more scintillation than would a normal stream, suggesting that a compression region moving at an intermediate velocity is present in the ray-path

data, though the velocities are most accurately determined from the best fit to the scattering model. These results indicate an undisturbed slow stream at $380 \mathrm{~km} \mathrm{~s}^{-1}$ and a "fast" stream that is considerably slower and produces much more scintillation than expected. We are confident that the observations of $1229+020$ on 5 September 1991 show a compressed interaction region at the leading edge of the fast stream.

The observation shown in Fig. 8 was at a radial distance of $87 R$, but intermediate velocities associated with fast-stream/slow-stream interaction regions have been observed when the ray-path has passed as close to the Sun as $27 R$. Figure 9a shows a white-light map centred on 24 August 1994: the superimposed white lines are the lines of sight for EISCAT observations of $1042+120$ on 24 August 1994, projected down onto 


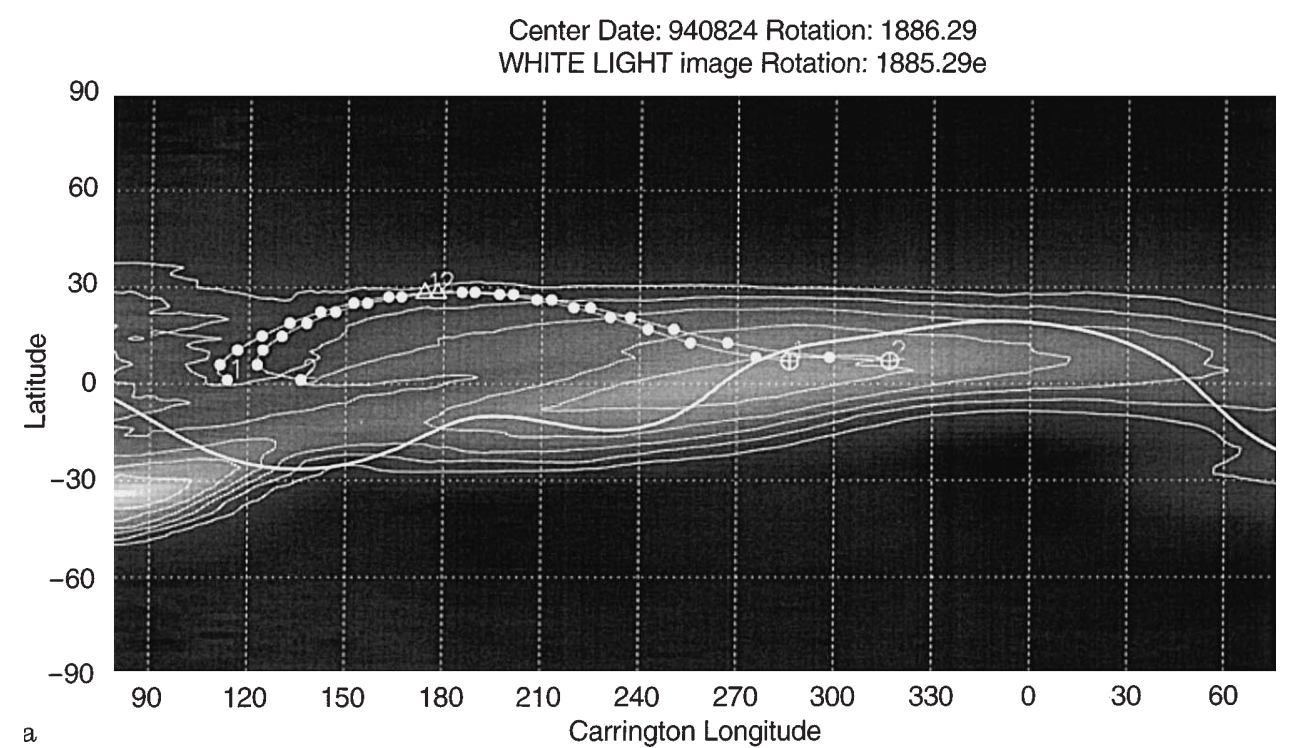

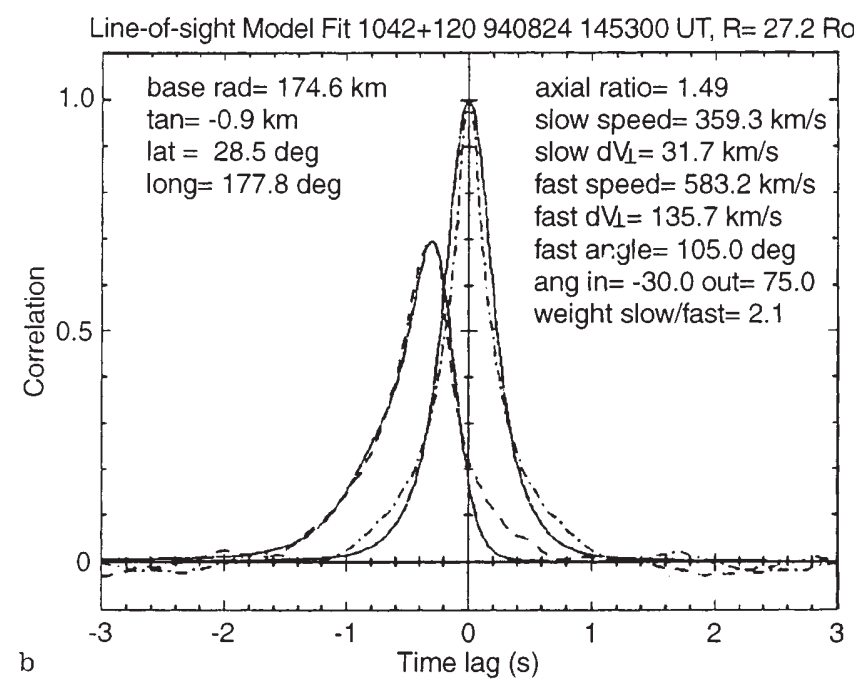

Fig. 9. a White-light map showing the ray-path for an EISCAT observation of $1042+120$ on 24 August 1994, projected down to 1.7 $R$ with assumed velocities of $750 \mathrm{~km} \mathrm{~s}^{-1}$ (line 1) and $325 \mathrm{~km} \mathrm{~s}^{-1}$ (line 2). The white line stretching across the whole of the map is the magnetic equator, calculated from Stanford magnetic data. b EISCAT correlation functions for the observation shown in a. The

the corona with assumed velocities of 800 (line 1) and $400 \mathrm{~km} \mathrm{~s}^{-1}$ (line 2). Figure 9b shows the corresponding correlation functions. The results of the scattering model suggest that the ray-path includes a normal slow stream with a velocity of about $360 \mathrm{~km} \mathrm{~s}^{-1}$, but the "fast" stream is much slower than normal and produces much more scintillation. These results are very similar to those shown in Fig. 8 and strongly suggest that CIRs may begin to develop inside $30 \mathrm{R}$. This result was most unexpected as we had not expected to see any evidence of interaction so close to the Sun. However, modelling of the interaction region (see Sect. 4.1) has shown that significant compression can develop inside $30 R$. We believe that our observations show the intermediate velocity flow in the dense, compressed region lying across the stream interface. observed correlation functions are shown as broken lines and the results of the model fit as solid lines. The "fast" component is slower and produces more scintillation than would a normal stream, suggesting that a compression region moving at an intermediate velocity is present in the ray-path

\subsection{Intermediate velocities and CIRs}

During the 1995-1997 campaigns 25 observations showed evidence of velocities intermediate between fast and slow streams (when intermediate velocities are defined as more than two standard deviations away from the mean velocities of streams that are expected to be more than $97.5 \%$ fast or $97.5 \%$ slow). As discussed in Sect. 1, four of these streams could be associated with CMEs seen in LASCO or Mk.3 white-light data, leaving 21 intermediate velocities not associated with any obvious transient event. The white-light maps corresponding to these observations were examined to see if the geometry of fast and slow streams would place a compression region in the ray-path. In most cases it was quite clear whether an interaction region should be 
present in the ray-path or not, but there were a number of ambiguous cases when the ray-path lay along the border of a coronal hole. It was necessary to resort to a degree of subjective judgement in classifying these events. The results for the 22 intermediate velocities are summarised in Table 2.

An alternative approach is to determine (from the white-light maps) which observations have fast and slow streams in the correct orientation for the compression region to lie in the ray-path. By the criteria illustrated in Fig. 7 there are 32 observations highly likely to include a compression region. By classifying velocities more than $2 \sigma$ away from the means of fast or slow velocities as intermediate, velocities more than $1.75 \sigma$ but less than $2.0 \sigma$ away from each mean as "probably intermediate" and those between $1.5 \sigma$ and $1.75 \sigma$ away as "possibly intermediate", it is possible to compare the expected occurrence of compression regions with observations of intermediate velocities.

The results for these 32 observations are summarised in Table 3. Twenty of these observations showed clear intermediate velocities and nine showed evidence of "probable" or "possible" intermediate velocity flow, as defined in the last paragraph. This is strong evidence that the intermediate velocity flows observed by EISCAT are associated with regions of interaction between the fast and slow solar winds.

Three observations showed clear fast and slow streams with no evidence of any intermediate velocity. However, all of these measurements were made very close to the Sun - inside $22 R$ - and so lay close to or inside the inner limit for weak scattering. Observations of compression regions close to the Sun are discussed in Sect. 3.4.

There are a few remaining difficulties in determining the points of origin of streams of solar wind. In particular, the white-light maps derived from Mk.3 coronagraph measurements correspond to a distance of $1.7 R$ from the centre of the Sun. At this distance from the Sun the solar wind has not reached its final velocity; moreover, the fast streams are expanding into the regions above the bright corona. These problems could be resolved by using data from the LASCO C3 coronagraph to generate white-light maps corresponding to distances of $8 R$ or more from the centre of the Sun, by which time the flow is close to radial and the acceleration of the fast wind complete.

\subsection{Repeated observations of intermediate velocity regions}

If the intermediate velocity regions observed in IPS measurements correspond to the compression regions at the leading edge of CIRs, they should be expected to recur at intervals of the solar rotation period.

The data set used in this study includes five clear observations of features which were seen on two successive solar rotations. An example from May/June 1997 is shown in Fig. 10. Parts a and b of Fig. 10 show white-light coronal maps centred on 22 May and 16 June 1997. The IPS lines of sight superimposed on the maps correspond to observations of (a) $0431+206$ on 22 May and (b) $0603+219$ on 16 June. In each case lines 1 and 2 represent the IPS ray-path projected down onto the corona with assumed velocities of 750 and $325 \mathrm{~km} \mathrm{~s}^{-1}$, respectively. The IPS correlation functions for these two observations are shown in Fig. 10c and d, respectively.

The form of the low-latitude coronal holes seen in Fig. 10a and $\mathrm{b}$ are very similar, suggesting that the same feature is being observed on two successive rotations. On each occasion the geometry is correct to place a

Table 2. Summary of 21 intermediate velocities observed between April 1995 and July 1997. These observations were made when the geometry of the IPS ray-path was likely to place a compression region within the field of view of the observation

\begin{tabular}{|c|c|c|c|c|c|c|c|c|c|}
\hline $\begin{array}{l}\text { Date } \\
\text { yymmdd }\end{array}$ & source name & $\theta_{\text {in }}$ & $\theta_{\text {out }}$ & comment & $\begin{array}{l}\text { distance } \\
(R)\end{array}$ & lat. & $\operatorname{limb}$ & $\begin{array}{l}V_{\text {fast }} \\
\mathrm{km} \mathrm{s}^{-1}\end{array}$ & $\begin{array}{l}V_{\text {slow }} \\
\mathrm{km} \mathrm{s}^{-1}\end{array}$ \\
\hline 950526 & $0318+164$ & -40 & 0 & CIR? & 48 & -1 & W & 578 & 383 \\
\hline 950601 & $0318+164$ & -60 & -10 & CIR? & 61 & 1 & W & 563 & 382 \\
\hline 950609 & $0431+206$ & -45 & 0 & CIR & 32 & -1 & W & 562 & 287 \\
\hline 950703 & $0738+177$ & -10 & 50 & CIR? & 47 & -23 & $\mathrm{E}$ & 590 & - \\
\hline 950704 & $0738+177$ & -20 & 40 & CIR & 44 & -24 & $\mathrm{E}$ & 578 & 350 \\
\hline 950906 & $1150-003$ & -20 & 30 & CIR & 64 & -3 & $\mathrm{E}$ & 559 & 310 \\
\hline 950924 & $1256-057$ & -10 & 20 & CIR & 52 & 4 & $\mathrm{E}$ & 550 & 297 \\
\hline 960522 & $0403+260$ & -50 & 80 & CIR? & 28 & -17 & $\mathrm{E}$ & 509 & 241 \\
\hline 960817 & $0854+201$ & 0 & 30 & CIR & 54 & 14 & $\mathrm{~W}$ & 590 & 401 \\
\hline 960823 & $1042+120$ & -60 & 0 & CIR & 29 & 27 & $\mathrm{E}$ & 573 & 241 \\
\hline 960915 & $1118+125$ & -60 & 60 & CIR & 39 & 46 & W & 587 & 346 \\
\hline 960919 & $1256-057$ & -70 & -10 & CIR & 68 & 4 & $\mathrm{E}$ & 522 & 187 \\
\hline 970616 & $0603+219$ & -20 & 40 & CIR & 22 & -22 & $\mathrm{E}$ & 577 & 363 \\
\hline 970628 & $0521+166$ & -10 & 20 & CIR? & 63 & -14 & W & 548 & 296 \\
\hline 970629 & $0604+203$ & -20 & 25 & CIR & 27 & -19 & W & 588 & 288 \\
\hline
\end{tabular}


Table 3. Summary of probable compression regions in EISCAT IPS data, 1995-1997 (as determined from white-light data). In each case the velocity of the dominant stream is shown in bold type.
Cases when intermediate velocities were not observed are shown in italics. $D(V)$ is the difference in standard deviations of the velocity of the dominant stream from the mean fast or slow flow speed

\begin{tabular}{|c|c|c|c|c|c|c|c|c|c|}
\hline Date & source & $\theta_{\text {in }}$ & $\theta_{\text {out }}$ & $\begin{array}{l}\text { dist } \\
(R)\end{array}$ & lat. & $\operatorname{limb}$ & $\begin{array}{l}V_{\text {fast }} \\
\mathrm{km} \mathrm{s}^{-1}\end{array}$ & $\begin{array}{l}V_{\text {slow }} \\
\mathrm{km} \mathrm{s}^{-1}\end{array}$ & $D(V)$ \\
\hline 950528 & $0318+164$ & -10 & 30 & 55 & 0 & W & 563 & 382 & 2.6 \\
\hline 950609 & $0431+206$ & -45 & 0 & 31 & -1 & W & 562 & 287 & 2.6 \\
\hline 950704 & $0738+177$ & -20 & 40 & 44 & -24 & $\mathrm{E}$ & 578 & 350 & 2.4 \\
\hline 950705 & $0738+177$ & -20 & 30 & 41 & -25 & $\mathrm{E}$ & 562 & 443 & 2.6 \\
\hline 950906 & $1150-003$ & -20 & 30 & 64 & -3 & $\mathrm{E}$ & 559 & 310 & 2.7 \\
\hline 950907 & $1042+120$ & -60 & 0 & 28 & 28 & W & 617 & 296 & 1.8 \\
\hline 950924 & $1256-057$ & -10 & 20 & 52 & 4 & $\mathrm{E}$ & 550 & 297 & 2.8 \\
\hline 960522 & $0431+206$ & -20 & 10 & 28 & -17 & $\mathrm{E}$ & 509 & 241 & 3.4 \\
\hline 960523 & $0431+206$ & -40 & 10 & 25 & -28 & $\mathrm{E}$ & 639 & 242 & 1.6 \\
\hline 960524 & $0431+206$ & -15 & 15 & 21 & -20 & $\mathrm{E}$ & 619 & 258 & 1.8 \\
\hline 960525 & $0431+206$ & -50 & 10 & 18 & -23 & $E$ & 745 & 413 & 1.1 \\
\hline 960823 & $1042+120$ & -60 & 0 & 29 & 27 & $\mathrm{E}$ & 573 & 241 & 2.5 \\
\hline 960824 & $1042+120$ & -70 & -10 & 26 & 30 & $\mathrm{E}$ & 621 & - & 1.8 \\
\hline 960915 & $1118+125$ & -60 & 60 & 39 & 46 & $\mathrm{~W}$ & 587 & 346 & 2.3 \\
\hline 960915 & $1120+143$ & -60 & 50 & 45 & 50 & $\mathrm{~W}$ & 632 & 319 & 1.6 \\
\hline 960919 & $1256-057$ & -70 & -10 & 68 & 4 & $\mathrm{E}$ & 522 & 197 & 3.0 \\
\hline 970520 & $0431+206$ & -20 & 30 & 36 & -14 & $\mathrm{E}$ & 548 & 331 & 2.8 \\
\hline 970522 & $0431+206$ & -20 & 30 & 29 & -16 & $\mathrm{E}$ & 562 & 482 & 2.6 \\
\hline 970522 & $0521+166$ & -25 & 40 & 74 & -25 & $\mathrm{E}$ & 693 & 513 & 2.4 \\
\hline 970523 & $0431+206$ & -40 & 0 & 26 & -17 & $\mathrm{E}$ & 590 & 429 & 2.3 \\
\hline 970524 & $0431+206$ & -40 & 0 & 22 & -19 & $E$ & 681 & 349 & 0.99 \\
\hline 970525 & $0431+206$ & -40 & 40 & 19 & -21 & $E$ & 735 & 298 & 0.18 \\
\hline 970605 & $0431+206$ & -10 & 50 & 20 & -6 & $\mathrm{~W}$ & 449 & 301 & 4.2 \\
\hline 970616 & $0603+219$ & -20 & 40 & 22 & -22 & $\mathrm{E}$ & 577 & 363 & 2.4 \\
\hline
\end{tabular}

compression region in the ray-path and clear intermediate velocities are observed.

These observations provide strong evidence that the intermediate velocities seen by IPS are associated with long-lived (more than 27 days) features in the corona. Unfortunately EISCAT did not make IPS observations during July of 1997, so we are unable to determine whether this feature would have remained detectable over three rotations.

\subsection{CIRs close to the Sun}

Spacecraft observations have studied the development of CIRs out to over $1000 R$, but to date no in situ measurements have been made closer to the Sun than 70 $R$. At this distance Helios showed that CIRs were already well developed, although the reverse and forward shocks of the "classical" interplanetary CIR did not form until distances greater than $215 R$ (e.g. Hu, 1993).

IPS measurements are uniquely well suited to making measurements of the solar wind over a wide range of distances from the Sun and so are particularly useful for studying the evolution of the solar wind.

The inner limit for our line-of-sight integration analysis is imposed by the onset of strong scattering, typically at 15 solar radii in the fast wind and 25-30 solar radii in the slow wind. In the strong scattering regime the incident and scattered waves interact and the resultant destructive interference reduces the scintillation power received on Earth. The transition from weak to strong scattering is therefore marked by a "turnover" in the scintillation power. The power received increases as the Sun is approached up until the onset of strong scattering and then falls away. Thus, by considering the variation of scintillation power from a given source as it approaches the Sun, we can determine when the observations are from regions of weak scattering and so can be reliably interpreted.

Figure 11 shows IPS observations of the radio source $0431+206$ as it moved in towards the Sun. In all cases the geometry of the ray-path would place a compression region in the IPS field of view, as shown in the whitelight maps (Fig. 11a-c ). The correlation functions from these observations are shown in Fig. 11d-f. Finally, the scintillation power measured at the two sites is plotted against the distance of closest approach of the ray-path to the Sun (Fig. 11g).

The observations from 22 May 1996, when the closest approach of the ray-path to the Sun was $27.9 R$, show a clear intermediate velocity and significantly enhanced scintillation from the "fast" stream (Fig. 11a). The 

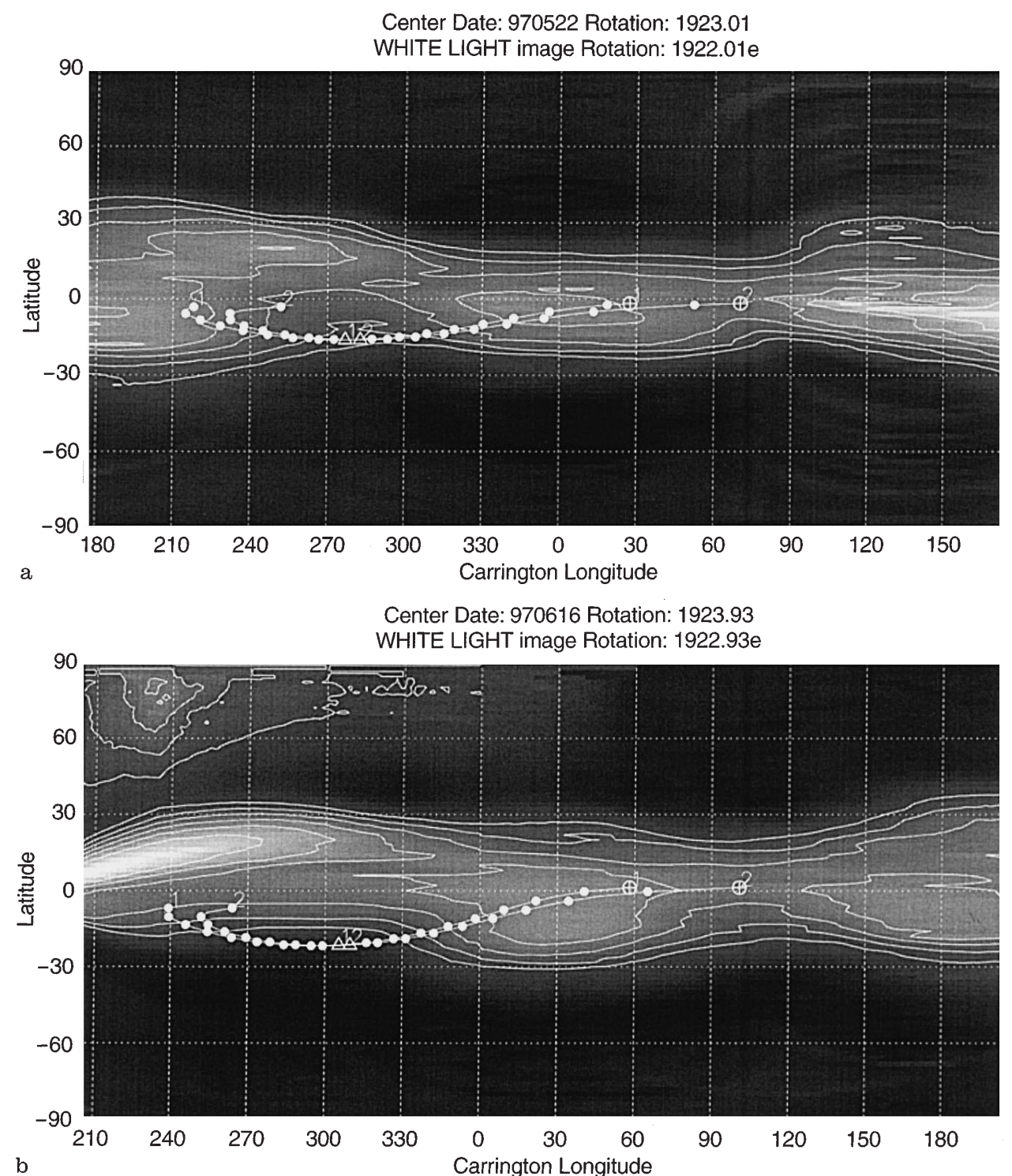

b

Line-of-sight Model Fit 0431+206970522 121600 UT, $R=29.4$ Ro

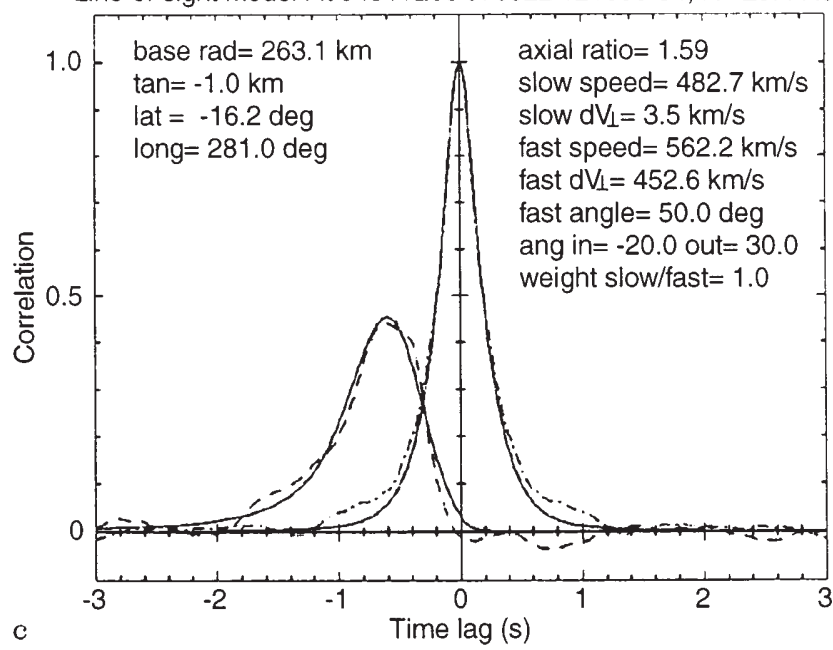

Fig. 10. a White-light coronal map centred on 22 May 1997. The lines superimposed on the map represent the IPS ray-path for an observation of $0431+206$, projected down to $1.7 R$ with assumed velocities of $750 \mathrm{~km} \mathrm{~s}^{-1}$ (line 1) and $325 \mathrm{~km} \mathrm{~s}^{-1}$ (line 2). b White-light coronal map centred on 16 June 1997. The lines superimposed on the map represent the IPS ray-path for an observation of $0603+219$, projected down to
Line-of-sight Model Fit 0603+219 970616094600 UT, R=21.5 Ro

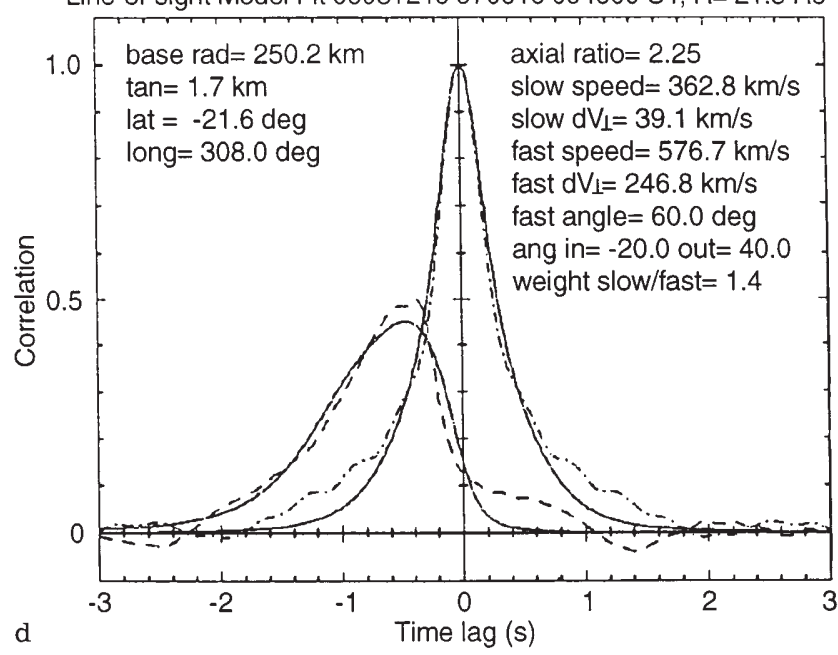

$1.7 R$ with assumed velocities of $750 \mathrm{~km} \mathrm{~s}^{-1}$ (line 1) and $325 \mathrm{~km} \mathrm{~s}^{-1}$ (line 2). c Correlation functions for the observation of $0431+206$ on 22 May 1997 shown in part a. d Correlation functions for the observation of $0603+219$ on $16^{\text {th }}$ June 1997 shown in part b. The observed correlation functions in $\mathbf{c}$ and $\mathbf{d}$ are shown as broken lines and the results of the model fit as solid lines 
Center Date: 960522 Rotation: 1909.63

WHITE LIGHT image Rotation: $1908.63 \mathrm{e}$

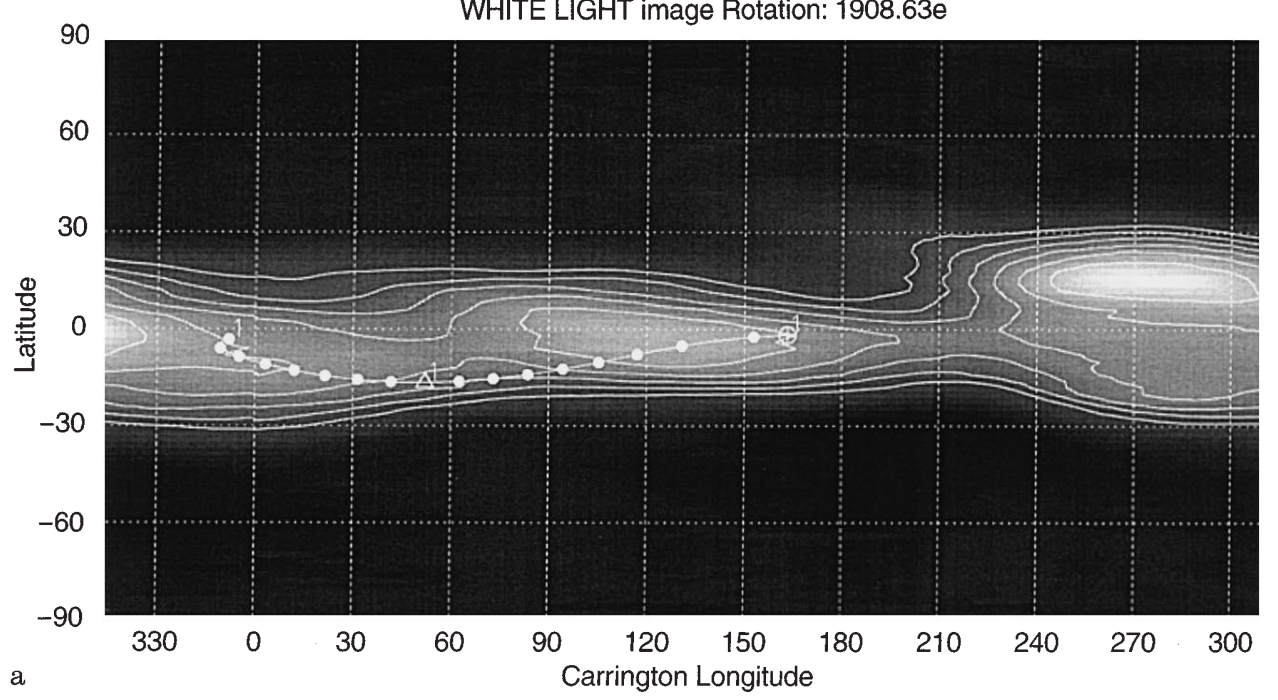

Center Date: 960523 Rotation: 1909.67 WHITE LIGHT image Rotation: 1908.67e

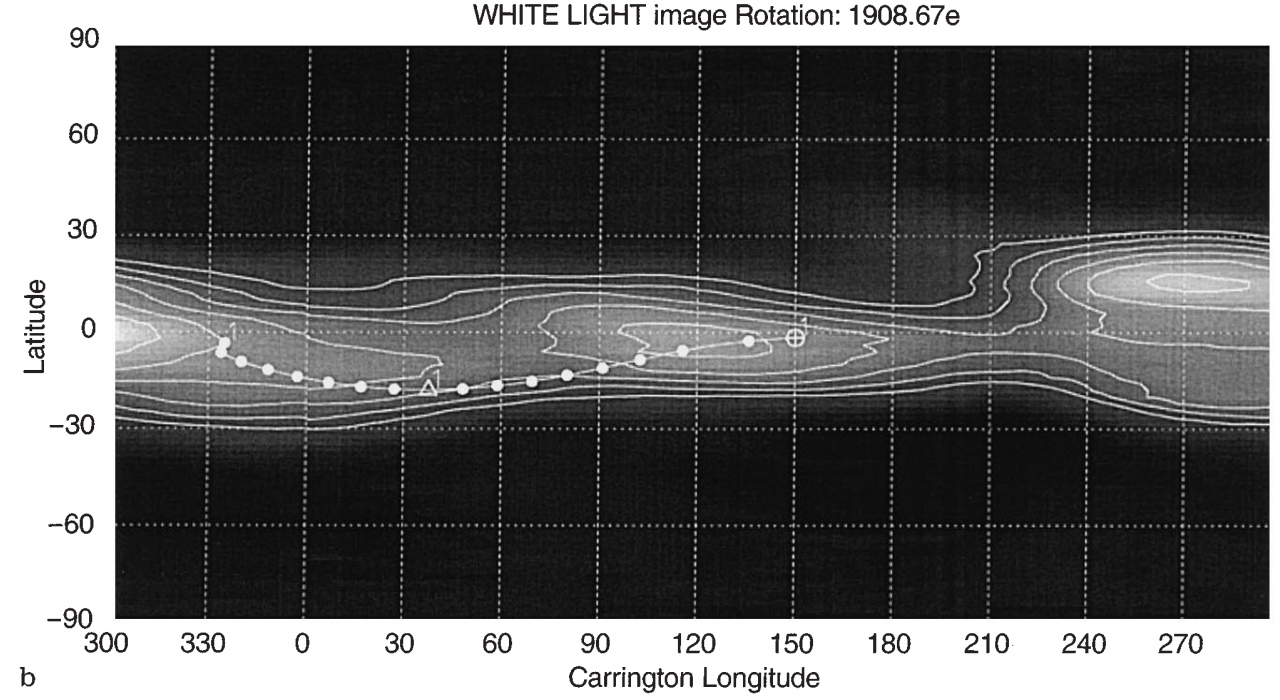

Center Date: 960525 Rotation: 1909.74 WHITE LIGHT image Rotation: $1908.74 \mathrm{e}$

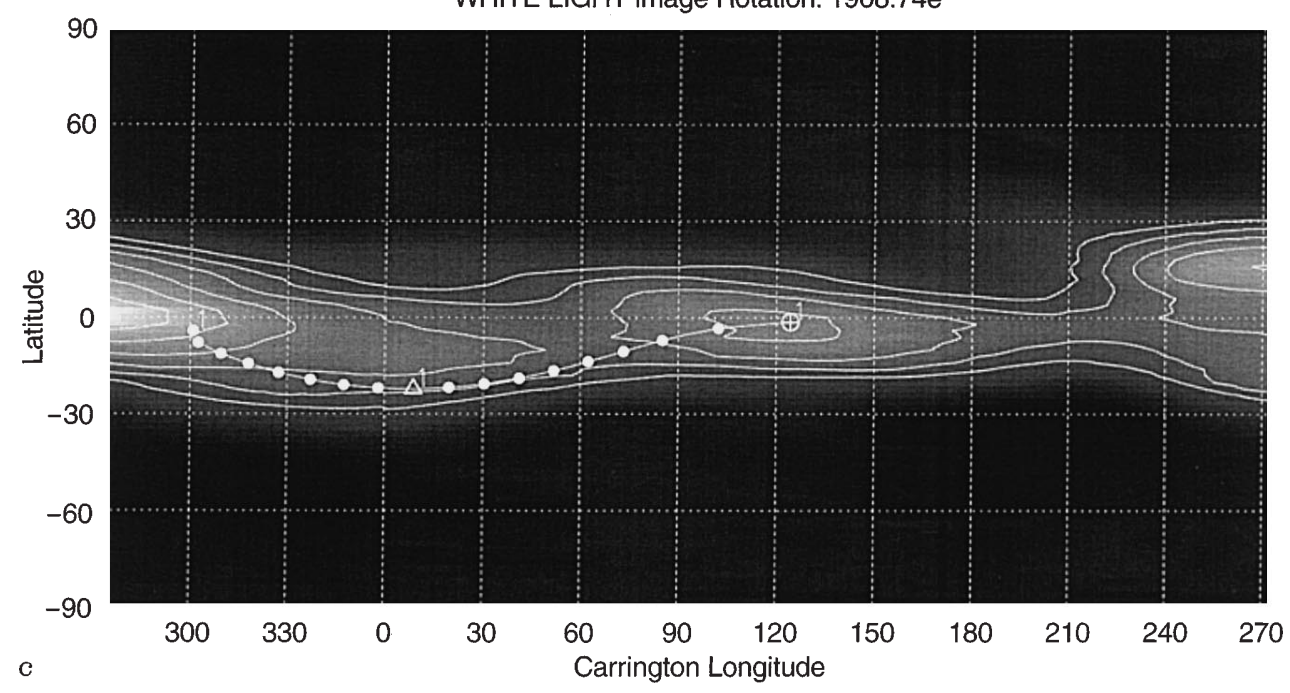

Fig. 11. a-c 

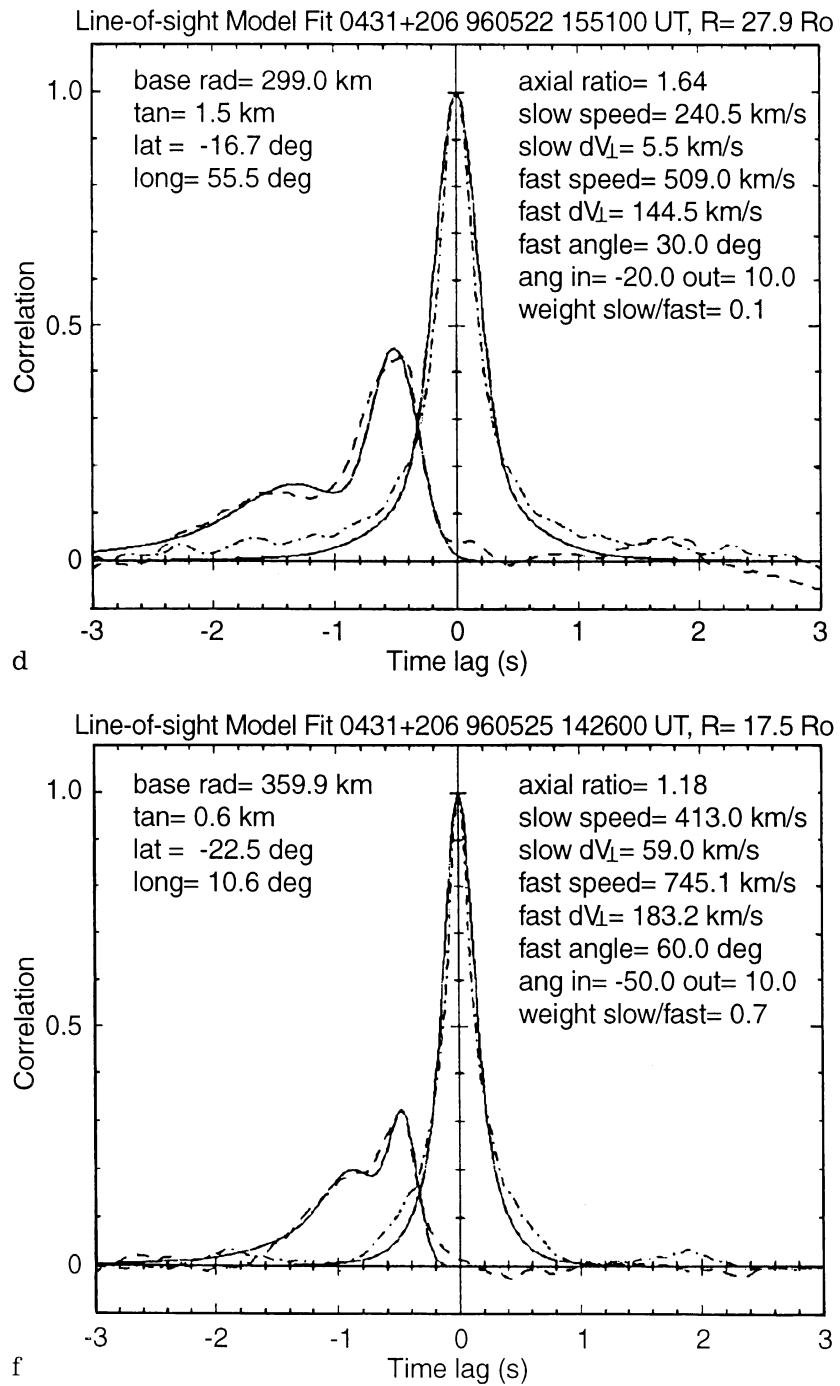

Fig. 11. a White-light map of the coronaat $1.7 R$, centred on 22 May 1996. The IPS ray-path for the observation of $0431+206$ at $27.9 R$ is shown projected down to $1.7 R$ with an assumed velocity of $750 \mathrm{~km} \mathrm{~s}^{-1}$. In this figure (and all subsequent white-light maps) the line corresponding to a projection velocity of $325 \mathrm{~km} \mathrm{~s}^{-1}$ is omitted for clarity. b White-light map of the corona at $1.7 R$, centred on 23 May 1996. The IPS ray-path for the observation of $0431+206$ at 24.6 $R$ is shown projected down to $1.7 R$ with an assumed velocity of $750 \mathrm{~km} \mathrm{~s}^{-1}$. c White-light map of the corona at $1.7 R$, centred on 25 May 1996. The IPS ray-path for the observation of $0431+206$ at 17.9 $R$ is shown projected down to $1.7 R$ with assumed velocities of $750 \mathrm{~km} \mathrm{~s}^{-1}$. d Correlation functions from the observation of $0431+206$ on 22 May 1996. The observed correlation functions (in parts d, e and f) are shown as broken lines and the results of the model

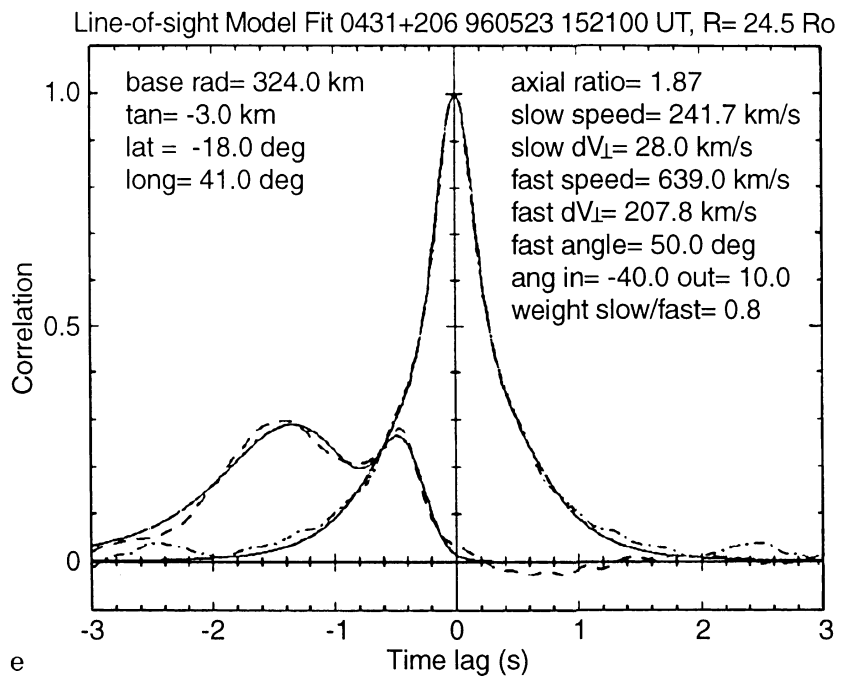

0431+206, 1996/05/22 - 1996/05/25

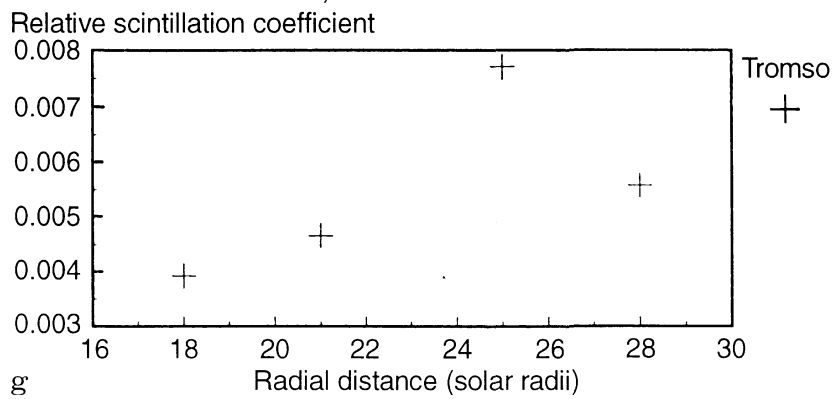

fit as solid lines. A dense region moving at an intermediate velocity is present in the ray-path. e Correlation functions from the observation of $0431+206$ on 23 May 1996. The observation lies on the boundary between weak and strong scattering, but the results suggest that a region of intermediate velocity flow may be present. f Correlation functions from the observation of $0431+206$ on $25^{\text {th }}$ May 1996. The ray-path passes through a region of strong scattering. Clear fast and slow components are present. g Relative scintillation index observed by EISCAT (Troms $\phi$ site) plotted against radial distance of the point of closest approach of the ray-path to the Sun (in solar radii). The raypath passes into strong scattering when its closest approach to the Sun lies within $25 R$. The relative scintillation index is defined as the RMS variation of the observed power divided by the mean observed power

normal fast stream. An intermediate velocity was still detectable in the correlation functions measured on 24 May 1997, when the compression region was $21.3 R$ from the Sun but by the next day clear fast and slow streams were present, with no evidence of intermediate velocity flow (Fig. 11f). However, the ray-path had passed into strong scattering when the distance of closest approach became less than about $24 R$ : this renders interpretation of these later results difficult and, as yet, it is impossible to determine whether the observations from 21 and $18 R$ indicate that the 
interaction region starts to develop at about $20 R$ or that the onset of strong scattering makes it impossible to detect compression regions closer to the Sun than this distance. The observations from 22 May are clearly in the weak scattering regime and show convincing evidence of a compression region present in the ray-path at only $28.3 R$ from the Sun. We believe this to be the closest to the Sun that an interaction region has been detected using any technique.

These observations are not unique in the EISCAT data set, and Fig. 12 shows a similar set of measurements from May 1997. The radio source is again $0431+206$ and intermediate velocities continue to be observed after the onset of strong scattering inside $26 R$ (Fig. $12 \mathrm{c}, \mathrm{d}, \mathrm{e})$. A clear intermediate velocity was seen at $29.4 R$, corresponding to a compression region which was inside $30 R$ from the Sun (shown in Fig. 10c). The results of these two series of observations are summarised in Table 4.

\section{Discussion}

IPS observations have detected regions of enhanced scintillation moving at velocities intermediate between those of fast and slow streams. All observations of
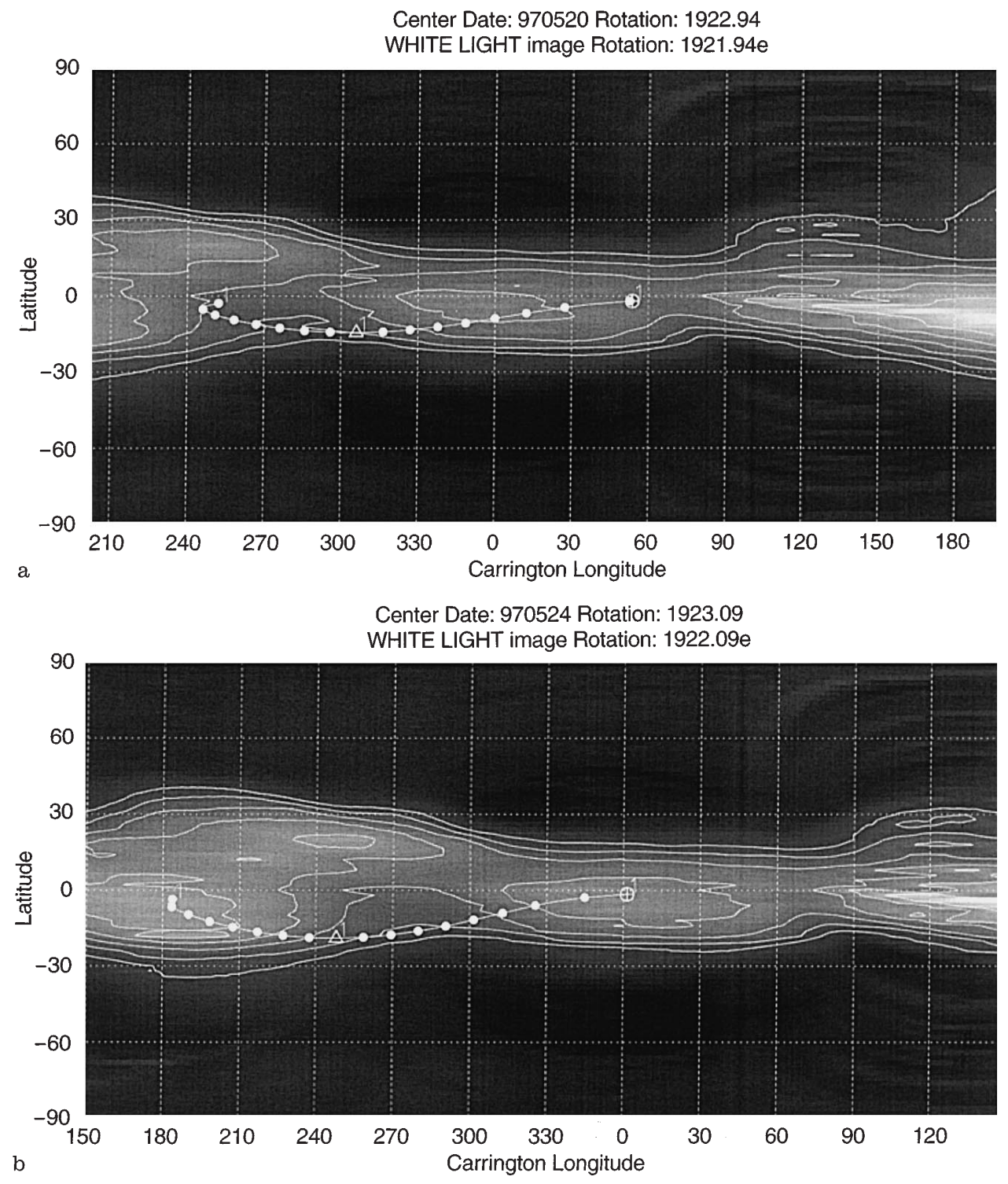

Fig. 12. a White-light map of the corona at $1.7 R$, centred on 20 May 1997. The IPS ray-path for the observation of $0431+206$ at $36 R$ is shown projected down to $1.7 R$ with assumed velocities of $750 \mathrm{~km} \mathrm{~s}^{-1}$. b White-light map of the corona at $1.7 R$, centred on 24 May 1997. The IPS ray-path for the observation of $0431+206$ at $22 R$ is shown projected down to $1.7 R$ with assumed velocities of $750 \mathrm{~km} \mathrm{~s}^{-1}$. c Correlation functions from the observation of $0431+206$ on 20 May 1997. A dense region moving at an intermediate velocity is present in the ray-path. d Correlation functions from the observation of $0431+206$ on $24^{\text {th }}$ May 1997. In c and $\mathbf{d}$ the observed correlation functions are shown as broken lines and the results of the model fit as solid lines. The ray-path passes through a region of strong scattering. Clear fast and slow components are present. e Relative scintillation index observed by EISCAT (Kiruna site) plotted against radial distance of the point of closest approach of the ray-path to the Sun (in solar radii). The ray-path passes into strong scattering when its closest approach to the Sun lies within $30 R$ 


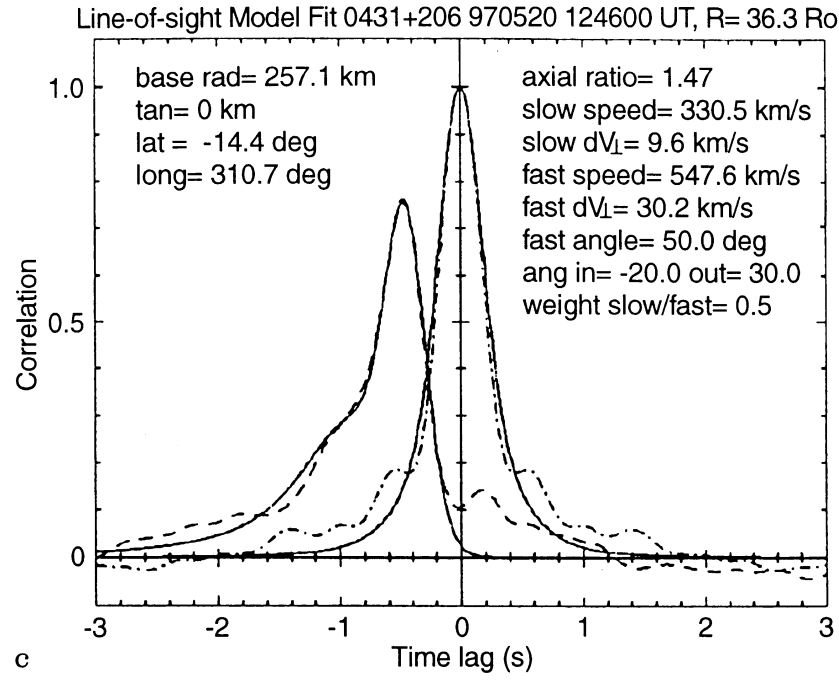

Relative scintillation coefficient

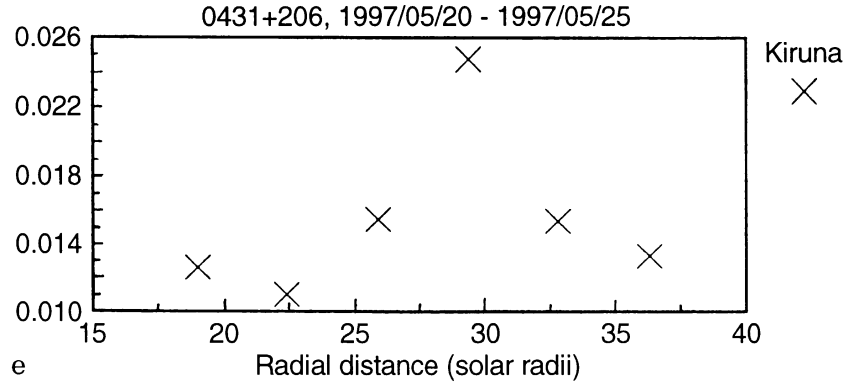

intermediate velocities occur either during the passage of a CME or when the geometry of the observation would place the compression zone of CIR in the IPS ray-path. The observations described in Sect. 3.3 have shown that these intermediate velocities re-occur at intervals comparable to the solar rotation period and appear to be associated with long-lined low-latitude coronal holes seen in white-light data. We therefore have no hesitation in associating the majority of intermediate velocities observed by EISCAT with CIRs between fast and slow streams of solar wind.

Table 4. Compression regions observed close to the Sun. Asterisks denote observations which include regions dominated by strong scattering. In all cases the "fast" stream density is the average

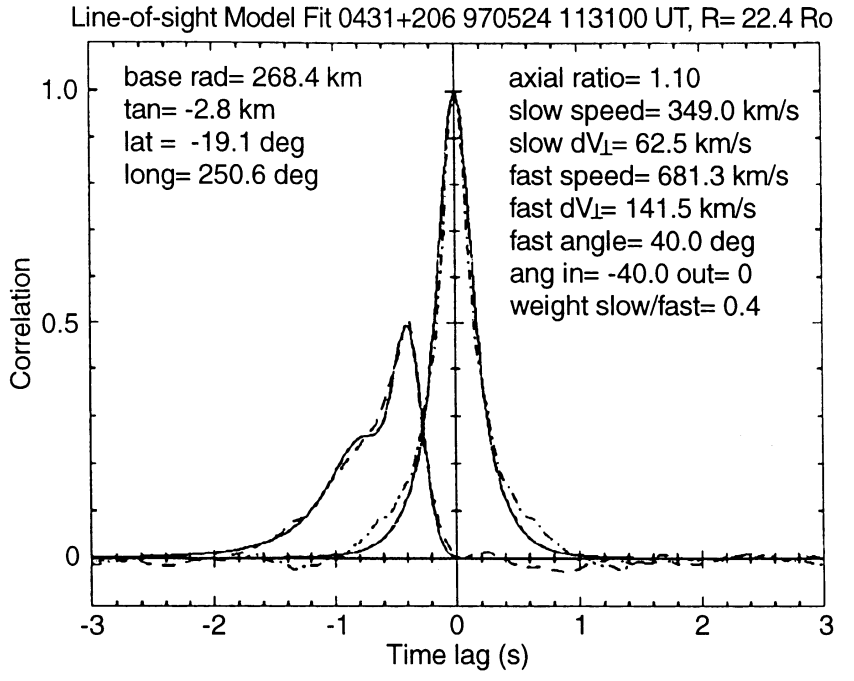

d

Fig. 12. c-e.

\subsection{Early development of CIRs}

EISCAT observations indicate that compression regions at the leading edge of fast streams are already well developed by 26-28 $R$. At these distances from the Sun the angle between the magnetic fields carried by the fast and slow winds is small. Furthermore, a significant compression can only be produced when the speed difference between the interacting streams is greater than the speed of the waves which transport information through the plasma.

density of the fast stream and the compression region: the density of the compression region will be susbstantially higher

\begin{tabular}{|c|c|c|c|c|c|c|}
\hline Date & source & $\begin{array}{l}\text { distance of } \\
\text { closest approach } \\
(R)\end{array}$ & $\begin{array}{l}\text { Distance of inferred } \\
\text { compression region } \\
(R)\end{array}$ & $\begin{array}{l}V_{\text {fast }} \\
\mathrm{km} \mathrm{s}^{-1}\end{array}$ & $\begin{array}{l}V_{\text {slow }} \\
\mathrm{km} \mathrm{s}^{-1}\end{array}$ & $\begin{array}{l}\text { density } \\
\text { fast:slow }\end{array}$ \\
\hline 960522 & $0431+206$ & 27.9 & 28.3 & 509 & 241 & 7.1:1 \\
\hline 960523 & $0431+206$ & 24.6 & 25.0 & 639 & 242 & $1.2: 1$ \\
\hline $960524 *$ & $0431+206$ & 21.0 & 21.3 & 619 & 250 & $1.8: 1$ \\
\hline 970520 & $0431+206$ & 36.0 & 41.6 & 548 & 331 & $1.9: 1$ \\
\hline 970522 & $0431+206$ & 29.0 & 33.5 & 562 & 483 & $2.0: 1$ \\
\hline $970523 *$ & $0431+206$ & 26.0 & 26 & 590 & 429 & $1.4: 1$ \\
\hline $970524 *$ & $0431+206$ & 22 & 22 & 681 & 349 & $2.3: 1$ \\
\hline $970525^{*}$ & $0431+206$ & 19 & 24 & 735 & 298 & $1.6: 1$ \\
\hline
\end{tabular}


Modelling of wave speeds for fast, slow, and intermediate modes can be achieved to a good approximation outside $30 R$, as one can assume that the relevant physical parameters are smoothly varying beyond the acceleration region. Values of temperature, magnetic field strength and alignment, particle density, and speed can be obtained either from spacecraft or EISCAT measurements. The resulting wave speeds are shown to be already less than the speed difference between the interacting streams by as little as $30 R$. For this reason, we can state that the potential for interaction exists by $30 R$, although the degree of interaction will increase as one looks further away from the Sun. It has yet to be established how the interaction potential changes with time, as this would indicate over which length scale the proto-interactions have the potential to steepen into CIRs.

\subsection{Solar wind velocities in the ecliptic plane}

EISCAT observations of the solar wind at distances between 15 and $100 R$ show that it is clearly dominated

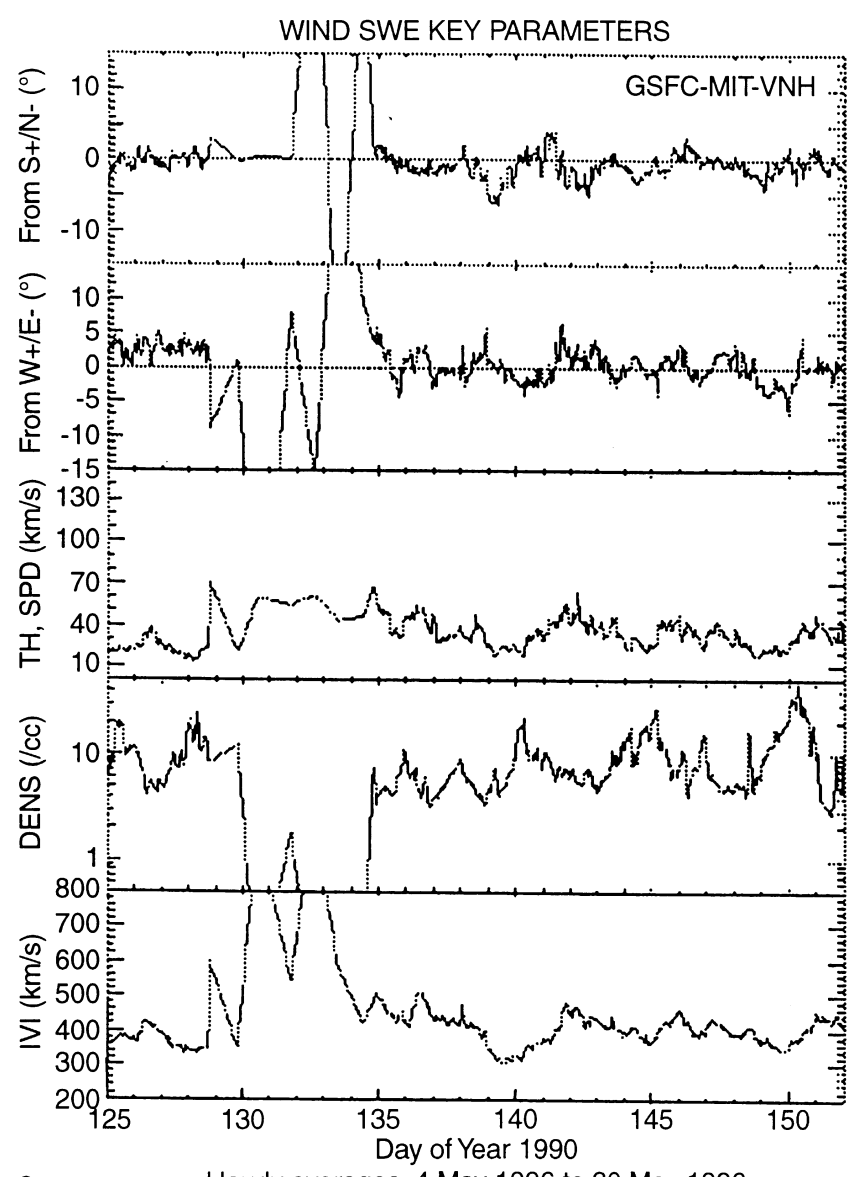

a

Haurly averages: 4 May 1996 to 30 May 1996

Fig. 13. a Measurements of solar wind parameters at 1 AU $(215 R)$ from the Wind spacecraft, 4 May 1996-30 May 1996. The compression region observed by EISCAT on 22 May 1996 intersected the orbit of the Wind spacecraft between day 149 and day 151 of the year-29 (30 May), when Wind detected the intermediate flow velocities $\left(\sim 450 \mathrm{~km} \mathrm{~s}^{-1}\right)$ and enhanced densities characteristic of an by fast streams with scintillation velocities of about $750 \mathrm{~km} \mathrm{~s}^{-1}$ and slow streams with velocities of about $325 \mathrm{~km} \mathrm{~s}^{-1}$ and relatively few velocities in the 500-650$\mathrm{km} \mathrm{s}^{-1}$ range. These results are in good agreement with Ulysses observations from a wide range of latitudes, suggesting that the difference between scintillation velocity and bulk plasma velocity is small. However, a large quantity of spacecraft data from $215 R(1 \mathrm{AU})$ in the ecliptic plane show "fast stream" velocities of around $500-600 \mathrm{~km} \mathrm{~s}^{-1}$ (e.g. Fig. 13a,b). At solar minimum coronal holes penetrating towards the equator are generally narrow (e.g. the "Elephant's Trunk" coronal hole seen in carrington Rotations 1912 and 1913, which covered approximately $15^{\circ}$ of longitude at the heliographic equator), making it probable that fast streams from these features would have merged with their interaction regions by $1 \mathrm{AU}$. Apart from observations made during the passage of a coronal mass ejection, EISCAT observations of intermediate velocities are clearly associated with fast-stream/slow-stream interaction regions. Close to the Sun these compression regions are only a few degrees wide (Schwenn, 1990) but expand rapidly beyond $70-80 R$ to effect longitude

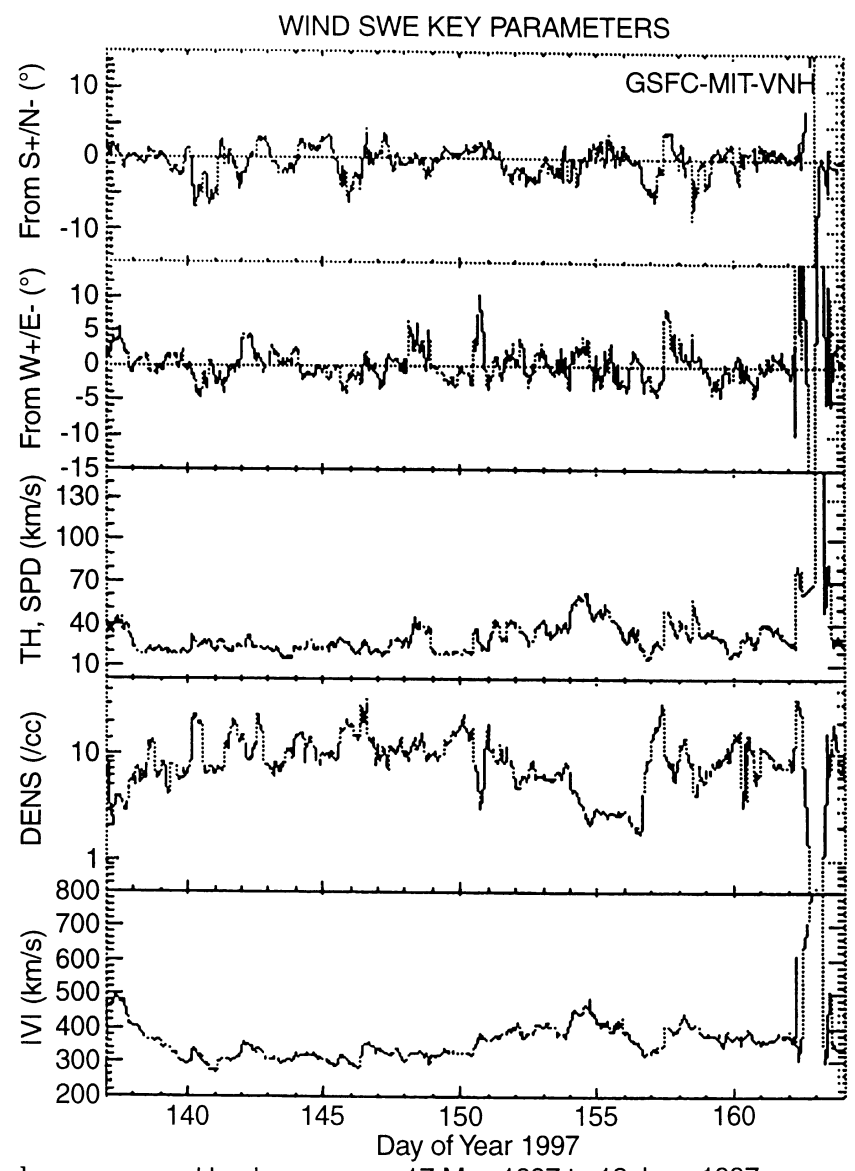

b

interaction region. b Measurements of solar wind parameters at $1 \mathrm{AU}$ (215 R) from the Wind spacecraft, 17 May 1996-12 June 1997. The compression region observed by EISCAT on 23 May 1997 intersected the orbit of the Wind spacecraft between day 150 and day 151 of the year (30-31 May) when Wind detected intermediate flow velocities $\left(\sim 440 \mathrm{~km} \mathrm{~s}^{-1}\right)$ and enhanced densities 
ranges of $70^{\circ}$ or more by $215 R$. By this time several corotating features may have merged, with the resultant CIR containing several stream interfaces (e.g. Schwenn, 1990). We suggest that the "fast streams" of $\sim 500$ $600 \mathrm{~km} \mathrm{~s}^{-1}$ observed by spacecraft in the ecliptic plane are actually the merged remnants of one or more narrow fast streams originating near the solar equator. By $1 \mathrm{AU}$ these fast streams have lost their identity, having been "swallowed up" by the expanding compression region.

At $525 \mathrm{~km} \mathrm{~s}^{-1}$, a packet of solar wind plasma will take around $68 \mathrm{~h}$ to travel from the observed interaction regions at 26-30 $R$ to Earth orbit. The IPS observations are of regions above the east limb of the Sun, so it is also necessary to take account of the time taken for the Sun to rotate through $90^{\circ}$, carrying the co-rotating feature seen on the east limb round to spacecraft close to the Earth. Taken together, these two effects mean that corotating features should be seen by a spacecraft close to the Earth about 9-10 days after they appear in IPS measurements from $30 R$ over the east limb of the Sun.

Measurements from the Wind spacecraft show increased plasma velocities from 29-31 May (days 150152 of year), when the interaction region observed by EISCAT on 22 May 1996 was in line with the Earth (Fig. 13a). The velocities are of the order of 450 $480 \mathrm{~km} \mathrm{~s}^{-1}$, significantly faster than the normal slow wind and well within the range of intermediate velocities observed by EISCAT. In each case the decrease in density and the increase in flow speed occurs in the Wind data slightly before the time of passage of the stream interface: this is consistent with the model predictions published by Pizzo (1989, 1991), which showed the compression region forming just ahead of the stream interface. The Wind observations are given (in italics) in Table 4.

The results from the 1997 event are equally clear, with a significant enhancement in flow speed and a smaller increase in density on days 149-151 (29-31 May), when the interaction region observed by EISCAT on 23-24 May would have intersected the orbit of Wind. These results are shown in Fig. 13b.

We therefore suggest that the velocities of 500 $600 \mathrm{~km} \mathrm{~s}^{-1}$ frequently observed by spacecraft at Earth orbit are the merged remains of narrow fast streams and their compression regions.

\section{Conclusions}

IPS observations from EISCAT have shown that away from interaction regions and transients the solar wind consists of clear fast and slow components. The fast streams are now known to originate in coronal holes, which makes it possible to use white-light and soft X-ray solar images to determine which regions of the IPS raypath lie in fast flow and which in the denser slow wind. This in turn makes it possible to determine the true velocities and densities of the fast and slow streams.

A minority of EISCAT IPS observations show velocities intermediate between those of fast and slow streams. Of 25 such events observed between 1995 and 1997, 21 came from regions in which a fast stream was overtaking a slow stream and showed enhanced levels of scintillation from the intermediate velocity feature. We interpret these observations as compression regions at the leading edge of CIRs. The only intermediate velocities not associated with compression regions were observed during the passage of coronal mass ejections (Breen et al., 1997).

We have observed intermediate velocity events with characteristics consistent with observations of the compression region of CIRs as close to the Sun as $26 R-$ the closest to the Sun that such events have been seen.

Acknowledgements. We would like to thank the director and staff of EISCAT for the data used in this study. EISCAT is supported by the scientific research councils of Finland, France, Germany, Japan, Norway, Sweden and the UK. Three of us (ARB, PJM and CAV) were supported by PPARC during the period when this work was carried out.

Coronagraph data are used courtesy of the High Altitude Observatory, National Centre for Atmospheric Research (NCAR), Boulder, Cororado, U.S.A. NCAR is sponsored by the National Science Foundation.

The plots of Wind data are taken from the Wind WWW page at MIT (http://www.mit.edu:8001/afs/athena/org/s/space/www/ .html). We would like to thank K. J. W. Ogilvie, A. J. Lazarus and J. T. Steinberg for making this information available. We would also particularly like to thank A. P. Van Eyken for his cooperation in running the IPS experiment at EISCAT and E. Yasukawa and D. Koon for operating the Mk. 3 coronagraph.

The Editor-in-chief thanks E. A. Lucek and another referee for their help in evaluating this paper,

\section{References}

Armstrong, J., and W. A. Coles, Analysis of three-station interplanetary scintillation data, J. Geophys. Res, 77, 46024610, 1972.

Bourgois, G., W. A. Coles, G. Daign, J. Silen, T. Turenen, and P. J. S. Williams, Measurements of the solar wind velocity using EISCAT, Astron. Astrophys., 144, 452-462, 1985.

Breen, A. R., W. A. Coles, R. Grall, U-P. Løvhaug, J. Markkanen, H. Misawa, and P. J. S. Williams, EISCAT measurements of interplanetary scintillation, J. Atmos, Terr. Phys., 58, 507-519, 1996a.

Breen, A. R., W. A. Coles, R. R. Grall, M. T. Klinglesmith, J. Markkanen, P. J. Moran, B. Tegid, and P. J. S. Williams, EISCAT measurements of the solar wind, Ann. Geophysicae 14, 1235-1245, 1996b.

Breen, A. R., W. A. Coles, R. R. Grall, M. T. Klinglesmith, J. Markkanen, P. J. Moran, C. A. Varley, and P. J. S. Williams, EISCAT measurements of interaction regions in the solar wind, Adv. Space Res., 20, 27-30, 1997.

Burlaga, L. F., Interplanetary stream interfaces, J. Geophys. Res., 79, 3717-3725, 1974.

Coles, W. A., Interplanetary scintillation observations of the highlatitude solar wind, Space Sci. Rev., 72, 211-222, 1995.

Gosling J. T., A. J. Hundhausen, V. Pizzo, and J. R. Asbridge, Compressions and rarefactions in the solar wind: Vela 3, $J$. Geophys. Res., 77, 5442-5454, 1972.

Gosling, J. T., J. R. Asbridge, S. J. Bame, and W. C. Feldman, Solar wind stream interfaces, J. Geophys. Res., 83, 1401-1412, 1978.

Grall, R. R., W. A. Coles, M. T. Klinglesmith, A. R. Breen, and P. J. S. Williams, Rapid acceleration of the polar solar wind, Nature, 379, 429-432, 1996.

Hu, Y. Q., Evolution of corotating stream structures in the heliospheric equatorial plane, J. Geophys. Res., 98, 1320113214, 1993. 
Klinglesmith, M. T., The polar solar wind from 2.5 to 40 solar radii: results of intensity scintillation measurements, Ph.D. thesis, University of California, San Diego, 1997.

Krieger, A. S., A. F. Timothy, and E. C. Roelof, A coronal hole and its identification as the source of a high-velocity solar wind stream, Solar Phy. 23, 123, 1973.

Moran, P. J., A. R. Breen, C. A. Varley, P. J. S. Williams, W. P. Wilkinson, W. A. Coles, and J. Markkanen, measurements of the direction of the solar wind using interplanetary scintillation, Ann. Geophysicae, Ann. Geophysicae (this issue), 1998.

Neupert, W. M., and V. Pizzo, Coronal holes as sources of recurrent geomagnetic disturbances, J. Geophys. Res., 79, 37013709, 1974.

Nolte, J. T., J. M. Davis, M. Gerassimenko, A. J. Lazarus, and J. D. Sullivan, A comparison of solar wind speeds and coronal structure near solar minimum, Geophys. Res. Lett., 4, 291-294, 1977.

Phillips, J. L., A. Balogh, S. J. Bame, B. E. Goldsteen, J. T. Gosling, J. T. Hoeksema, D. J. McComus, M. Neugebauer, N. R. Sheeley, and Y. M. Wang, Ulysses at $50^{\circ}$ south: constant immersion in the high-speed solar wind, Geophys. Res. Lett., 12, 1105-1108, 1994.

Pizzo, V. J., 2D co-rotating stream fronts near the ecliptic plane in the inner solar wind, J. Geophys. Res., 94, 8673-8684, 1989.
Pizzo, V. J., The evolution of corotating stream fronts near the ecliptic plane in the inner solar system: 2. three-dimensional tilted-dipole fronts, J. Geophys. Res., 96, 5405-5420, 1991.

Rickett, B. J., and W. A. Coles, Evolution of the solar wind structure over a solar cycle: interplanetary scintillation velocity measurements compared with coronal observations, J. Geophys. Res., 96, 1717-1736, 1991.

Sarabhai, V., Some consequences of nonuniformity of solar wind velocity, J. Geophys. Res., 68, 1555-1557, 1963.

Schwenn, R., Large-scale structure of the interplanetary medium, in Physics of the inner Heliosphere I, Eds. R. Schwenn and E. Marsch, Springer, Berlin, Heidelberg, New York, pp.99-181, 1990.

Schwenn, R., H. Rosenbauer, H. Miggenrieder, and B. Meyer, Preliminary results of the Helios plasma experiment, In Procedings of the $18^{\text {th }}$ plenary meeting of COSPAR Varna, 1975, Ed. M.J. Rycroft, Space Res., 18, 1976.

Snyder, C. W., and M. Neugebauer, The relation of Mariner 2 plasma data to solar phenomena, in The solar wind, Eds. R.J. Mackin and M. Neugebauer, Pergamon Press, New York, pp. 25-34, 1966.

Wimmer-Schweingruber, R. F., R. von Steiger, R. Paerli, Solar wind stream interfaces in corotating interaction regions: SWICS/ Ulysses results, J. Geophys. Res., 102, 17407-17414, 1997. 